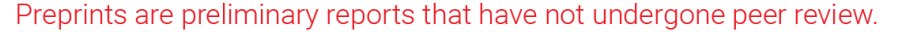 They should not be considered conclusive, used to inform clinical practice, or referenced by the media as validated information. \\ Design of SiN waveguides integrated with graphene oxide films for nonlinear optics
}

\section{Yang Qu}

Swinburne University of Technology

Jiayang Wu

Swinburne University of Technology

\section{Yuning Zhang}

Swinburne University of Technology

Linnan Jia

Swinburne University of Technology

Yao Liang

Swinburne University of Technology

\section{Baohua Jia}

Swinburne University of Technology

David Moss ( $\sim$ dmoss@swin.edu.au )

Swinburne University of Technology https://orcid.org/0000-0001-5195-1744

\section{Research Article}

Keywords: Four-wave mixing, 2D materials, silicon nitride, graphene oxide

Posted Date: April 16th, 2021

DOI: https://doi.org/10.21203/rs.3.rs-428184/v1

License: (c) (i) This work is licensed under a Creative Commons Attribution 4.0 International License.

Read Full License 


\title{
Design of SiN waveguides integrated with graphene oxide films for nonlinear optics
}

\author{
Yang Qu, Jiayang Wu, Member, IEEE, Yuning Zhang, Linnan Jia, Yao Liang, Baohua Jia, Fellow, \\ OSA, and David J. Moss, Fellow, IEEE, Fellow OSA
}

\begin{abstract}
We theoretically investigate and optimize four-wave mixing (FWM) in silicon nitride ( $\mathrm{SiN}$ ) waveguides integrated with two-dimensional (2D) layered graphene oxide (GO) films. Based on extensive previous measurements of the material parameters of the GO films, we perform detailed analysis for the influence of device parameters including waveguide geometry, GO film thickness, length, and coating position on the FWM conversion efficiency $(\mathrm{CE})$ and conversion bandwidth $(\mathrm{CB})$. The influence of dispersion and photo-thermal changes in the GO films is also discussed. Owing to the strong mode overlap between the SiN waveguides and the highly nonlinear GO films, FWM in the hybrid waveguides can be significantly enhanced. We obtain good agreement with previous experimental results and show that by optimizing the device parameters to balance the trade-off between Kerr nonlinearity and loss, the FWM CE can be improved by as much as $\sim 20.7 \mathrm{~dB}$ and the FWM CB can be increased by $\sim 4.4$ folds, relative to the uncoated waveguides. These results highlight the significantly enhanced FWM performance that can be achieved in SiN waveguides by integrating $2 D$ layered GO films.

Index Terms-Four-wave mixing, 2D materials, silicon nitride, graphene oxide.
\end{abstract}

\section{INTRODUCTION}

$\mathrm{F}$ our-wave mixing (FWM) is a parametric process that occurs in a nonlinear medium between incident light fields that interact to generate output light fields, potentially at new frequencies [1]. As a fundamental third-order $\left(\chi^{(3)}\right)$ nonlinear optical process $[2,3]$, it has found wide applications in alloptical signal generation, amplification, and processing such as wavelength conversion [2, 4], optical frequency comb generation [5-7], optical sampling [8, 9], quantum entanglement [10-13], and many others [14-17].

Implementing FWM devices in integrated platforms would provide significant advantages in terms of compact footprint, high stability, high scalability, and mass-producibility [18-20]. This, along with the ultrafast response time of FWM (typically on the order of $10^{-15} \mathrm{~s}$ [21]), can enable devices with ultrahigh processing speeds and ultralarge operation bandwidths. Despite the fact that silicon has been a leading integrated platform for many FWM devices [20, 22], its FWM performance is well known to be significantly limited by strong two-photon absorption (TPA) in the telecommunications band. Other complementary metal-oxide-semiconductor (CMOS) compatible platforms such as silicon nitride $(\mathrm{SiN})$ and doped silica $[19,23]$ have negligible TPA, although their Kerr

This work was supported by the Australian Research Council Discovery Projects Programs (No. DP150102972 and DP190103186), the Swinburne ECR-SUPRA program, the Industrial Transformation Training Centers scheme (Grant No. IC180100005), and the Beijing Natural Science Foundation (No. Z180007). (Corresponding author: Jiayang Wu, Baohua Jia, and David J. Moss) nonlinearity is only moderately higher than silica glass, thus representing a limitation in terms of FWM efficiency.

To improve the performance of the state-of-the-art integrated FWM devices, two-dimensional (2D) materials with an ultrahigh optical nonlinearity have been integrated onto chips to implement hybrid devices. Recently, hybrid integrated photonics devices incorporating graphene, graphene oxide (GO), black phosphorus, and transition metal dichalcogenides (TMDCs) with high FWM performance have been reported [24-28].

Benefiting from its ease of preparation as well as the tunability of its material properties, GO has been recognized as a promising 2D material $[29,30]$. Previously, GO films have been reported with a giant Kerr nonlinearity $\left(n_{2}\right)$ of about 5 orders of magnitude higher than that of $\operatorname{SiN}[31,32]$. The relatively large bandgap of $\mathrm{GO}(>2 \mathrm{eV}[29,33])$ results in a material absorption that is over 2 orders of magnitude lower than graphene as well as negligible TPA in the telecommunications band [26]. Low linear and nonlinear loss are highly desirable for nonlinear applications such as FWM. Based on these material properties, enhanced FWM in GOcoated $\mathrm{SiN}$ and doped silica waveguides has been demonstrated $[26,34]$. Another important advantage of GO is the ability to accurately control the film thickness, size and placement on integrated devices by using a large-area, transfer-free, layer-bylayer coating method together with standard lithography and lift-off processes [26, 35, 36]. This overcomes the limitations of mechanical layer transfer approaches that have been widely used for other 2D materials such as graphene and TMDCs [37] and enables cost-effective, large-scale, and highly precise integration of 2D layered GO films on a chip.

Previously [34], we experimentally demonstrated an enhancement of up to $\sim 9.1 \mathrm{~dB}$ in the FWM conversion efficiency (CE) of SiN waveguides integrated with layered GO films. We obtained extensive measurements of the loss and Kerr nonlinearity $\left(n_{2}\right)$ of the GO films that varied with film thickess and optical power. Here, we use these results to theoretically optimize the FWM performance of GO-coated

Yang Qu, Jiayang Wu, Yuning Zhang, Linnan Jia and D. J. Moss are with Optical Sciences Centre, Swinburne University of Technology, Hawthorn, VIC 3122, Australia. (e-mail: jiayangwu@ swin.edu.au; 1jia@swin.edu.au and dmoss@swin.edu.au).

Yao Liang and Baohua Jia are with Centre for Translational Atomaterials, Swinburne University of Technology, Hawthorn, VIC 3122, Australia. (e-mail; bjia@swin.edu.au). 
SiN waveguides. A detailed analysis of the influence of the device parameters, including waveguide geometry and GO film thickness, length, and coating position, on the FWM CE and conversion bandwidth (CB) is performed. The influence of dispersion and any photo-thermal changes in the GO films is also considered. We achieve good agreement with the previous experimental results and show that the enhancement in the FWM CE can be increased to as much as $\sim 20.7 \mathrm{~dB}$ and the FWM CB can be increased by $\sim 4.4$ folds, by optimizing the device parameters to balance the trade-off between Kerr nonlinearity and loss. These results highlight the strong potential for high FWM performance of SiN waveguides integrated with 2D layered GO films.

\section{DEVICE STRUCTURE}

Fig. 1(a) shows a schematic of a SiN waveguide coated with a patterned GO film, along with a schematic showing the atomic structure of GO with different oxygen functional groups (OFGs). In contrast to graphene that has a metallic behavior with zero bandgap [38], GO is a dielectric with a typical bandgap > $2 \mathrm{eV}[29,33]$ - much larger than what is expected for efficient TPA in the telecom band. SiN waveguides have been fabricated via low-pressure chemical vapor deposition (LPCVD) followed by lithography and dry etching processes, all of which are compatible with CMOS fabrication $[39,40]$. To enable film coating on the top surface of the SiN waveguides, the silica upper cladding is removed, typically via chemicalmechanical polishing (CMP). The coating of GO films can be achieved via a solution-based method, which yields layer-bylayer film deposition $[26,35,36]$. Compared with the complex layer transfer processes widely employed in coating other $2 \mathrm{D}$ materials such as graphene and TMDCs [24, 41, 42], this approach enables transfer-free coating of highly uniform films over large areas, with highly scalable fabrication processes as well as precise control of the number of layers (i.e., film thickness). Film patterning can be achieved using standard lithography and lift-off processes to achieve precise control of the film length and position [36] in order to optimize the FWM performance of the hybrid waveguides.

Fig. 1(b) shows the schematic cross section of the hybrid waveguide in Fig. 1(a). The corresponding transverse electric (TE) mode profile is shown in Fig. 1(c). Nonlinear optical processes such as FWM in the highly nonlinear GO film can be excited by the evanescent field of the waveguide mode, forming the basis for enhancing nonlinear optical performance of the hybrid waveguides.

Table I shows the definition of the parameters that we use to investigate FWM in the GO-coated $\mathrm{SiN}$ waveguides, including waveguide dimensions ( $W$ and $H)$, GO film parameters $(N, L$, and $\left.L_{0}\right)$, and continuous-wave (CW) optical powers $\left(P_{p}\right.$ and $\left.P_{s}\right)$. In the following sections, we first investigate the influence of mode overlap with the films, including the waveguide geometry ( $W$ and $H$ ) and film thickness $(N)$, on the loss and nonlinear parameter $(\gamma)$ of the hybrid waveguides. Next, by properly balancing the trade-off between loss and nonlinearity, we optimize the FWM CE in the hybrid waveguides for different waveguide geometries $(W, H)$ and GO film parameters $(N, L$,

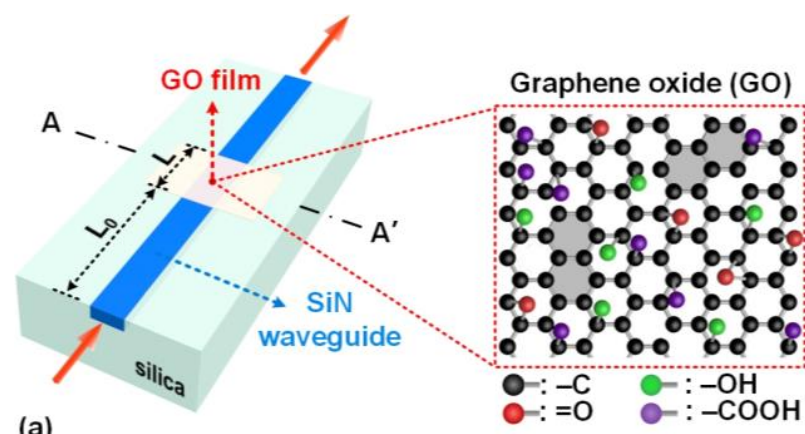

(a)

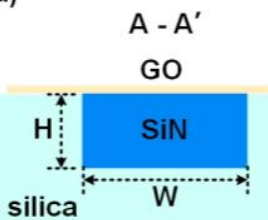

(b)

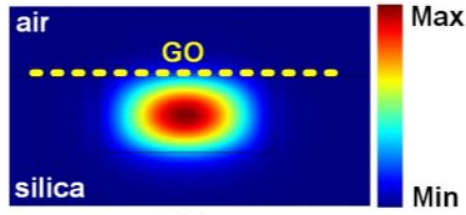

(c)
Fig. 1. (a) Schematic illustration of a SiN waveguide coated with 10 layers of GO. Inset shows schematics of atomic structure of GO. (b) Schematic illustration of the cross section of the hybrid waveguide in (a). (c) TE mode profile corresponding to (b). The definitions of $L_{0}, L, W$, and $H$ are given in Table I.

and $L_{0}$ ). Finally, we discuss the influence of the dispersion and any photo-thermal changes in the GO films in our model, as well as the influence of the film length on FWM CB and idler power evolution along the hybrid waveguides.

TABLE I. DEFINITIONS OF PARAMETERS OF WAVEGUIDES DIMENSION, GO FILM, AND CW LASER.

\begin{tabular}{|c|c|c|c|c|}
\hline $\begin{array}{l}\text { Waveguide } \\
\text { dimension }\end{array}$ & \multicolumn{2}{|c|}{ Height } & \multicolumn{2}{|c|}{ Width } \\
\hline Parameters & \multicolumn{2}{|l|}{$H$} & \multicolumn{2}{|c|}{$W$} \\
\hline GO film & $\begin{array}{c}\text { GO layer } \\
\text { number }\end{array}$ & \multicolumn{2}{|c|}{$\begin{array}{l}\text { Uncoated length } \\
\text { before GO segment }\end{array}$} & $\begin{array}{l}\text { Coating } \\
\text { length }\end{array}$ \\
\hline Parameters & $N$ & \multicolumn{2}{|c|}{$L_{0}$} & $L$ \\
\hline $\mathrm{CW}$ laser & \multicolumn{2}{|c|}{ Coupled pump power } & \multicolumn{2}{|c|}{ Coupled signal powe } \\
\hline Parameters & \multicolumn{2}{|c|}{$P_{p}$} & \multicolumn{2}{|c|}{$P_{s}$} \\
\hline
\end{tabular}

\section{LOSS \& NONLINEAR PARAMETER}

Mode overlap is a key factor that influences both loss and the nonlinear parameter $(\gamma)$ of GO-coated $\mathrm{SiN}$ waveguides. In this section, we investigate this using commercial mode solving softwares along with experimentally measured material parameters at $1550 \mathrm{~nm}$.

Fig. 2(a) shows the extinction coefficient $k$ of GO versus layer number $(N)$ for different input CW powers, which was obtained from our previous measurements [26, 36]. The $k$ of GO is over two orders of magnitudes lower than that of 

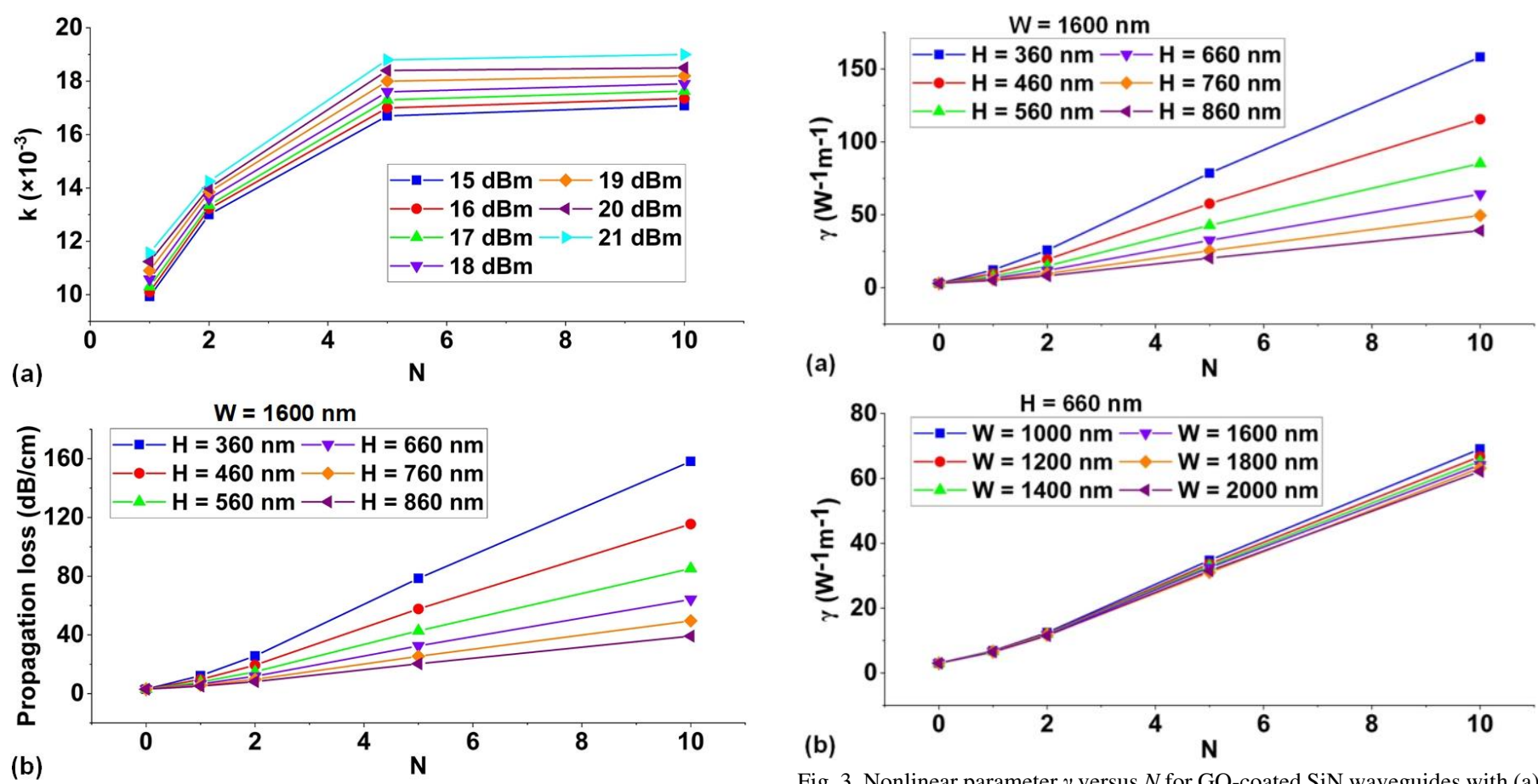

Fig. 3. Nonlinear parameter $\gamma$ versus $N$ for GO-coated $\mathrm{SiN}$ waveguides with (a) various $H$ when $W=1600 \mathrm{~nm}$ (b) various $W$ when $H=660 \mathrm{~nm}$. The points at $N=0$ correspond to the results for bare $\mathrm{SiN}$ waveguides.

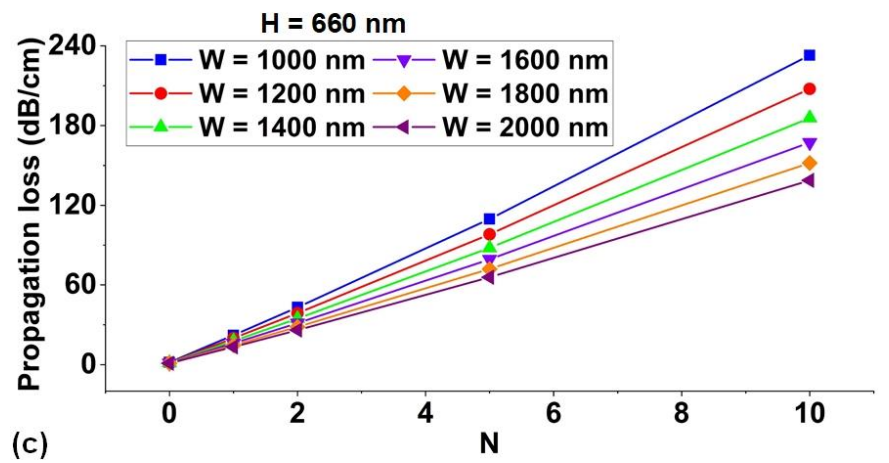

Fig. 2. (a) Extinction coefficient $k$ of GO versus $N$ under various input $\mathrm{CW}$ powers. (b) Propagation loss versus $N$ for GO-coated SiN waveguides with various $H$ when $W=1600 \mathrm{~nm}$. (c) Propagation loss versus $N$ for GO-coated SiN waveguides with various $W$ when $H=660 \mathrm{~nm}$. The points at $N=0$ correspond to the results for bare $\mathrm{SiN}$ waveguides.

graphene [43, 44], reflecting its potential for high-performance nonlinear photonic devices. The increase of $k$ with $N$ can be attributed to scattering loss stemming from film unevenness and imperfect contact between adjacent layers [26, 36]. The small but observable increase in $k$ with input $\mathrm{CW}$ power is mainly induced by power-dependent photo-thermal changes in the GO films [36, 45], which are not permanent and have a much slower time response than the nearly instantaneous nonlinear optical processes governing FWM and TPA [34, 46]. In contrast to $k$, the refractive index $n$ of GO showed negligible variation with $N$ and the input power [34], and so was taken as a constant (1.97) in our simulations.

Figs. 2(b) and (c) show the propagation loss of the hybrid waveguides versus GO layer number $(N)$, first for different waveguide heights $(H)$ with a fixed width $(W)$ and then for different widths $(W)$ with a fixed height $(H)$, calculated using Lumerical FDTD Mode Solutions. We used the experimentally measured values for the linear optical properties of both the GO films and the SiN waveguides, including the above $n, k$ of GO as well as previously fabricated $\mathrm{SiN}$ devices for the refractive

index $n$ of $\mathrm{SiN}$ (1.99) and propagation loss of the bare waveguides $(3.0 \mathrm{~dB} / \mathrm{cm})$ [34]. Note that the propagation loss is higher than buried $\mathrm{SiN}$ waveguides $(0.6 \mathrm{~dB} / \mathrm{cm})$ due to residual roughness induced by CMP, exacerbated by the higher SiN air refractive index difference. According to our previous measurements [26, 36], the film thickness is assumed to be proportional to $N$, with a thickness of $2 \mathrm{~nm}$ for each layer. TE polarization was chosen because the in-plane (TE polarized) interaction between the evanescent field and the GO film is much stronger than the out-of-plane (TM polarized) interaction due to the large optical anisotropy of 2D materials [26, 37].

In Fig. 2(b), as the height of the waveguide $H$ increases, the mode overlap with the films decreases, resulting in a decrease in propagation loss. At the same time, the propagation loss increases with GO layer number $N$, reflecting an increase in mode overlap for the hybrid waveguides having thicker films. Similar to the trends in Fig. 2(b), the propagation loss in Fig. 2(c) decreases with the waveguide width $W$ and increases with GO layer number $N$, further confirming that the propagation loss increases with mode overlap with the GO films.

Figs. 3(a) and (b) show the nonlinear paremeter of the hybrid waveguides $\gamma$ versus GO layer number $(N)$ for different waveguide geometries $(H, W)$, calculated based on methods described elsewhere [26, 46, 47]:

$$
\gamma=\frac{2 \pi}{\lambda} \frac{\iint_{\mathrm{D}} n_{0}^{2}(x, y) n_{2}(x, y) S_{\mathrm{Z}}{ }^{2} d x d y}{\left[\iint_{\mathrm{D}} n_{0}(x, y) S_{\mathrm{z}} d x d y\right]^{2}}
$$

where $\lambda$ is the pump wavelength (i.e., $1550 \mathrm{~nm}$ ), $D$ is the integral domain of the optical fields over the material regions, $S_{z}$ is the time-averaged Poynting vector calculated using COMSOL Multiphysics, $n_{0}(x, y)$ and $n_{2}(x, y)$ are the linear and nonlinear refractive index profiles over the waveguide cross section, respectively. In Eq. (1), $\gamma$ is an effective nonlinear 


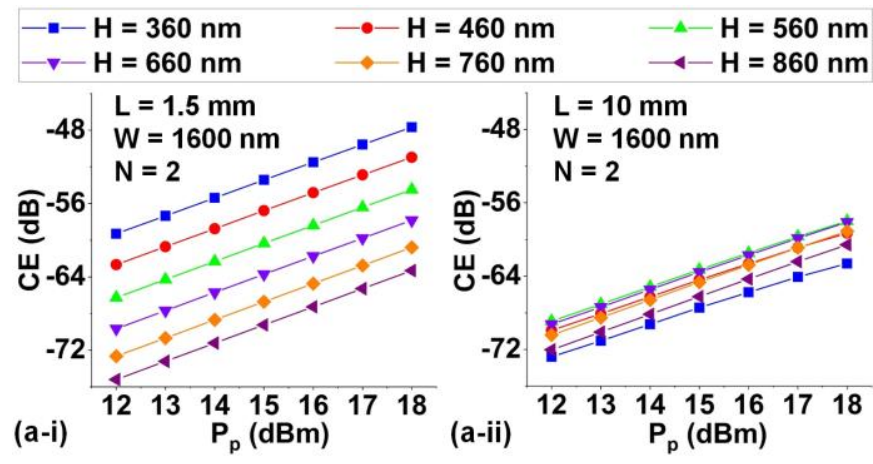

$-W=1000 \mathrm{~nm} \quad \longrightarrow-W=1200 \mathrm{~nm} \quad \triangle-W=1400 \mathrm{~nm}$
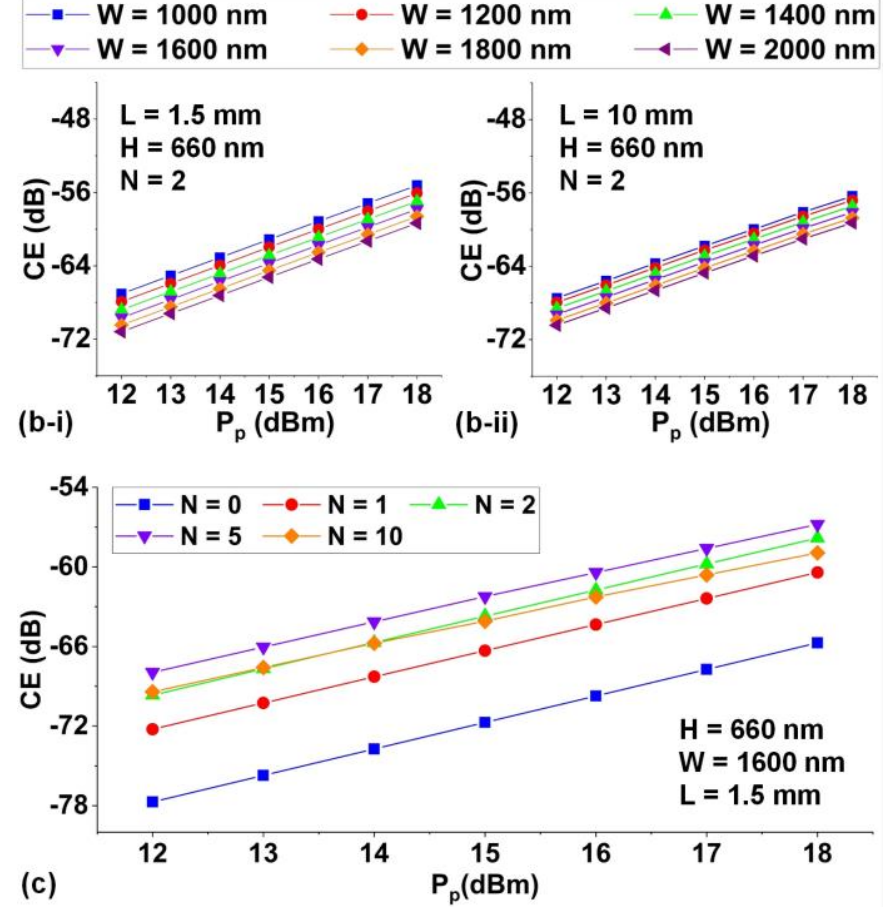

Fig. 4. FWM CE versus $P_{p}$ for GO-coated $\mathrm{SiN}$ waveguides with various (a) $H$, (b) W, and (c) $N$. In (a) and (b), $N=2$, (i) and (ii) show the results for $L=1.5$ $\mathrm{mm}$ and $L=10 \mathrm{~mm}$, respectively. In (a) and (c), $W=1600 \mathrm{~nm}$. In (b) and (c), $H=660 \mathrm{~nm}$. In (a) - (c), $L_{0}=0$.

parameter weighted not only by $n_{2}(x, y)$ but also by $n_{0}(x, y)$ in the different material regions. Note we used the Kerr nonlinearity $n_{2}(x, y)$ instead of the more general third-order nonlinearity $\left(\chi^{(3)}\right)$ because the FWM frequencies (pump, signal, idler) in our analysis are close enough together (with a pumpsignal wavelength detuning $<40 \mathrm{~nm}$ ) compared with any dispersion in $n_{2}$ [26, 48]. The values of $n_{2}$ for silica and $\mathrm{SiN}$ used in our calculations were $2.60 \times 10^{-20} \mathrm{~m}^{2} / \mathrm{W}$ [19] and 2.61 $\times 10^{-19} \mathrm{~m}^{2} / \mathrm{W}$ [34], respectively. The layer-dependent $n_{2}$ for GO was obtained from our previous experiments [34], which varied from $1.40 \times 10^{-14} \mathrm{~m}^{2} / \mathrm{W}$ for $N=1$ to $1.34 \times 10^{-14} \mathrm{~m}^{2} / \mathrm{W}$ for $N=$ 10 and were mainly induced by an increase in inhomogeneous defects and imperfect contact with film thickness. Since the experimentally measured $n_{2}$ of GO showed very little variation with optical power, we neglected this in our calculations.

In Figs. 3(a) and (b), we see that $\gamma$ increases with film thickness $N$ and decreases with waveguide height $H$ and width $W$, following the trends also seen with the propagation loss in Figs. 2(b) and (c). This indicates that increasing the mode
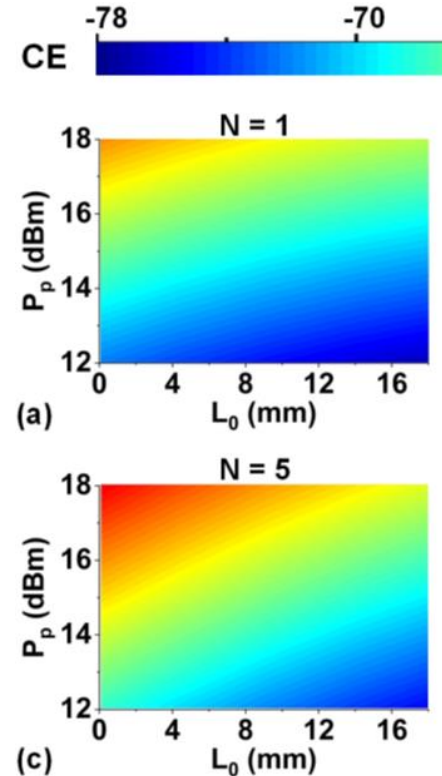

(b)
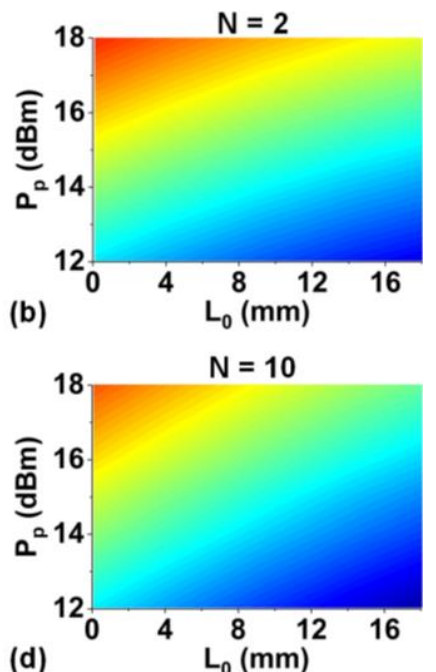

Fig. 5. FWM CE of GO-coated $\mathrm{SiN}$ waveguides versus $L_{0}$ and $P_{p}$ when (a) $N$ $=1$, (b) $N=2$, (c) $N=5$, and (d) $N=10$. In (a) - (d), $L=1.5 \mathrm{~mm}, H=660 \mathrm{~nm}$, and $W=1600 \mathrm{~nm}$.

overlap with GO increases both the Kerr nonlinearity and linear loss - a trade-off that needs to be properly balanced in order to optimize the nonlinear optical performance such as FWM.

\section{CONVERSION EFFICIENCY}

In this section, we investigate the influence of waveguide geometry $(W, H)$ and GO film parameters $\left(N, L\right.$, and $\left.L_{0}\right)$ on the FWM CE using the loss and $\gamma$ from section III. Considering only the idler at the shorter wavelength, and neglecting any depletion in the pump power due to idler generation, the FWM process in the GO-coated $\mathrm{SiN}$ waveguides can be modeled as $[49,50]$

$$
\begin{aligned}
\frac{d A_{p}(z)}{d z}= & -\frac{\alpha_{p}}{2} A_{p}(z)+j \gamma_{p}\left[\left|A_{p}(z)\right|^{2}+2\left|A_{s}(z)\right|^{2}+2\left|A_{i}(z)\right|^{2}\right] A_{p}(z) \\
& +j 2 \gamma_{p} A_{p}^{*}(z) A_{s}(z) A_{i}(z) \exp (j \Delta \beta z) \\
\frac{d A_{s}(z)}{d z}= & -\frac{\alpha_{s}}{2} A_{s}(z)+j \gamma_{s}\left[\left|A_{s}(z)\right|^{2}+2\left|A_{p}(z)\right|^{2}+2\left|A_{i}(z)\right|^{2}\right] A_{s}(z) \\
& +j \gamma_{s} A_{i}^{*}(z) A_{p}^{2}(z) \exp (-j \Delta \beta z) \\
\frac{d A_{i}(z)}{d z}= & -\frac{\alpha_{i}}{2} A_{i}(z)+j \gamma_{i}\left[\left|A_{i}(z)\right|^{2}+2\left|A_{p}(z)\right|^{2}+2\left|A_{s}(z)\right|^{2}\right] A_{i}(z) \\
& +j \gamma_{i} A_{s}^{*}(z) A_{p}^{2}(z) \exp (-j \Delta \beta z)
\end{aligned}
$$

where $A_{p, s, i}$ are the pump, signal, and idler amplitudes along the light propagation direction (i.e., $z$ axis), $\alpha_{p, s, i}$ are the linear losses in Fig. 2, $\gamma_{p, s, i}$ are the waveguide nonlinear parameters in Fig. 3, and $\Delta \beta=\beta_{s}+\beta_{i}-2 \beta_{p}$ is the linear phase mismatch, with $\beta_{p, s, i}$ denoting the propagation constants. In our case the wavelength detuning range was small $(\leq 40 \mathrm{~nm})$, and so the linear loss and nonlinear parameter can be assumed to be wavelength independent, i.e., $\alpha_{p}=\alpha_{s}=\alpha_{i}=\alpha, \gamma_{p}=\gamma_{s}=\gamma_{i}=\gamma$. By numerically solving Eqs. (2) - (4), the FWM CE was calculated according to 

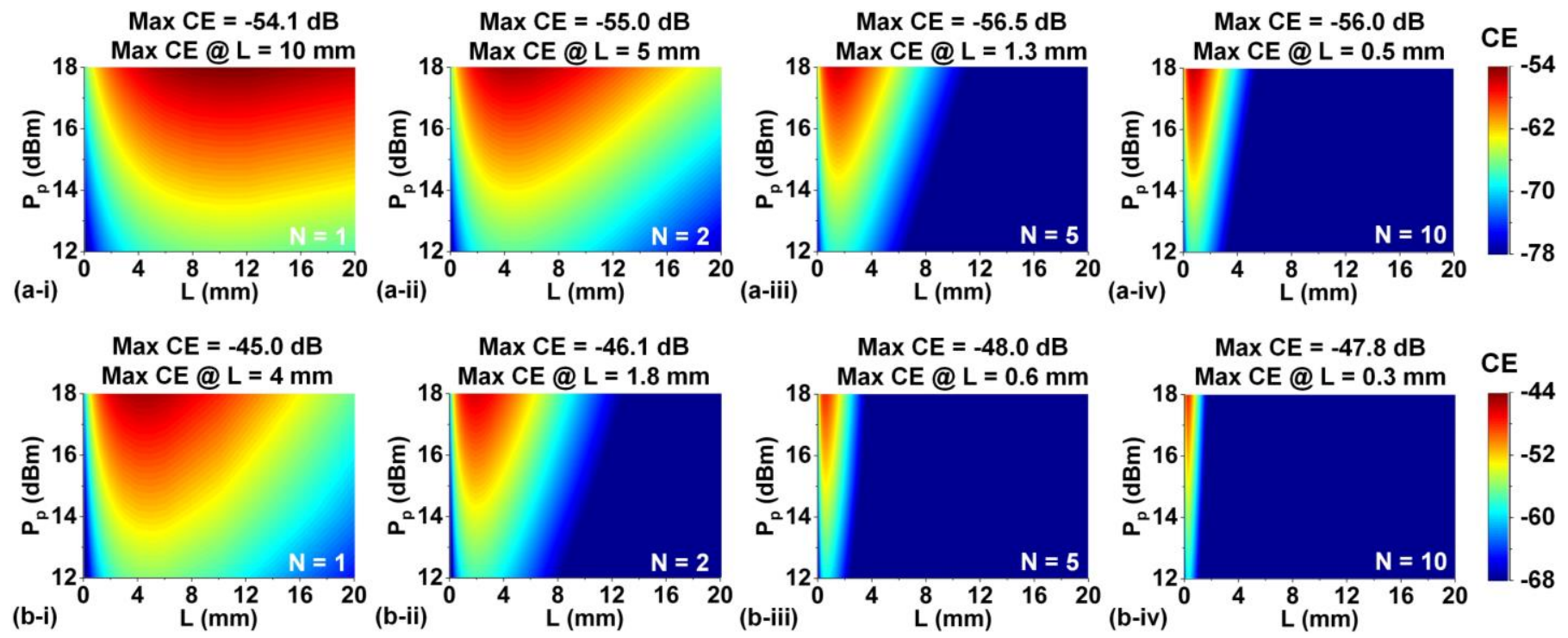

Fig. 6. FWM CE of GO-coated SiN waveguides versus $L$ and $P_{p}$ when (a) $W=1600 \mathrm{~nm}, H=660 \mathrm{~nm}$ and (b) $W=1000 \mathrm{~nm}, H=360 \mathrm{~nm}$. (i) - (iv) show the results for $N=1,2,5$, and 10, respectively. In (a) and (b), $L_{0}=0 \mathrm{~mm}$.

$$
C E(\mathrm{~dB})=10 \times \log _{10}\left[\left|A_{i}\left(L_{t}\right)\right|^{2} /\left|A_{s}(0)\right|^{2}\right]
$$

where $L_{t}$ is the total length of the $\mathrm{SiN}$ waveguide, taken as 20 $\mathrm{mm}$ in our analysis. The hybrid waveguides were divided into bare SiN (without GO films) and hybrid (with GO films) segments with different $\alpha, \gamma$, and $\beta_{p, s, i}$. Eqs. (2) - (4) were solved for each segment, and the output from the previous segment was used as the input for the subsequent segment.

In Figs. 4 (a) - (c), we compared the FWM CE of the hybrid waveguides while varying three parameters including the waveguide height and width $(H, W)$ and the GO film thickness $(N)$. In each figure, we varied only one parameter, keeping the other two constant. Fig. 4(a) compares the CE of the hybrid waveguides with the same width $W$ but different height $H$. The CE decreases with $H$ for short GO film lengths (i.e., $L=1.5 \mathrm{~mm}$ in Fig. 4(a-i)), reflecting that the increase in $\gamma$ dominates the trade-off between the Kerr nonlinearity and loss. Whereas for long GO film lengths (i.e., $L=10 \mathrm{~mm}$ in Fig. 4(a-i)), the CE varies non-monotonically with $H$, resulting in a maximum $\mathrm{CE}$ at an intermediate height $H=560 \mathrm{~mm}$. This is because the impact of loss becomes more prominent as $L$ increases. Fig. 4(b) compares the $\mathrm{CE}$ of the hybrid waveguides for a fixed height $H$ but different widths $W$. The CE decreases with $W$ monotonically for both $L=1.5 \mathrm{~mm}$ and $L=10 \mathrm{~mm}$, while the decrease becomes more gradual for $L=10 \mathrm{~mm}$. This reflects the fact that the CE is more sensitive to changes in $H$ rather than $W$, which is consistent with what is seen for the linear loss in Fig. 2 and $\gamma$ in Fig. 3. Fig. 4(c) compares the CE of the hybrid waveguides for different film thicknesses $N$. The maximum CE is also achieved at an intermediate $N=5$ as a result of the tradeoff between $\gamma$ and loss. In contrast to Figs. 4(a) and (b), the significant change in CE with $N$ results in an obvious trade-off between $\gamma$ and loss for a short GO film length of $L=1.5 \mathrm{~mm}$.

Figs. 5(a) - (d) show the CE of the hybrid waveguides versus coating position $L_{0}$ and pump power $P_{p}$ with $N=1,2,5$, and 10, respectively. For comparison, the other device parameters are kept the same as $L=1.5 \mathrm{~mm}, H=660 \mathrm{~nm}$, and $W=1600 \mathrm{~nm}$. In each figure, the CE increases with $P_{p}$ and decreases with $L_{0}$. The former reflects the increase in nonlinear efficiency with pump power while the latter results from a decrease in pump power in the GO-coated segment that dominates the FWM process in the hybrid waveguides. In contrast, for other waveguides than those considered here, such as for doped silica waveguides where the propagation loss of the bare waveguides is much lower, at $0.24 \mathrm{~dB} / \mathrm{cm}$ [26], the effect of varying the coating position $L_{0}$ would be much smaller.

Fig. 6(a) shows the $\mathrm{CE}$ of the hybrid waveguides versus film length $L$ and pump power $P_{p}$ with (i) $N=1$, (ii) $N=2$, (iii) $N=$ 5 , and (iv) $N=10$. For comparison, the other device parameters are kept the same as $L_{0}=0 \mathrm{~mm}, H=660 \mathrm{~nm}$, and $W=1600 \mathrm{~nm}$. In each figure, the $\mathrm{CE}$ increases with $P_{p}$, consistent with the trends seen in Fig. 5. The CE first increases with film length $L$ and then decreases, achieving maximum values at intermediate $L=10 \mathrm{~mm}, 5 \mathrm{~mm}, 1.3 \mathrm{~mm}$, and $0.5 \mathrm{~mm}$, respectively. This reflects the fact that the enhancement in the Kerr nonlinearity dominates for short film lengths while the loss dominates for long film lengths. As $N$ increases, the maximum CE shifts towards shorter lengths, reflecting the fact that the influence of loss increase becomes more significant for the hybrid waveguides having thicker GO films. The $\mathrm{CE}$ of the hybrid waveguide is $-56.6 \mathrm{~dB}$ when $N=5, L=1.5 \mathrm{~mm}$, and $P_{p}=18$ $\mathrm{dBm}$, which is $9.1 \mathrm{~dB}$ higher than comparable devices without GO films, showing a good agreement with our previous experimental results [34]. The maximum $\mathrm{CE}$ enhancement of $11.6 \mathrm{~dB}$ is achieved when $N=1, L=10 \mathrm{~mm}$, and $P_{p}=18 \mathrm{dBm}$, reflecting that there is still room for improvement in the experimental results by optimizing the GO film length and thickness.

Fig. 6(b) shows the corresponding results for hybrid waveguides with $H=360 \mathrm{~nm}$ and $W=1000 \mathrm{~nm}$ that have an enhanced mode overlap with GO films. The maximum CE of $45.0 \mathrm{~dB}$ is achieved when $N=1$ and $L=4 \mathrm{~mm}$, which is 20.7 $\mathrm{dB}$ higher than the bare waveguides and corresponds to $9.1 \mathrm{~dB}$ further improvement over the the maximum CE obtained in Fig. 6(a), reflecting the potential to improve the CE by optimizing the waveguide geometry. 

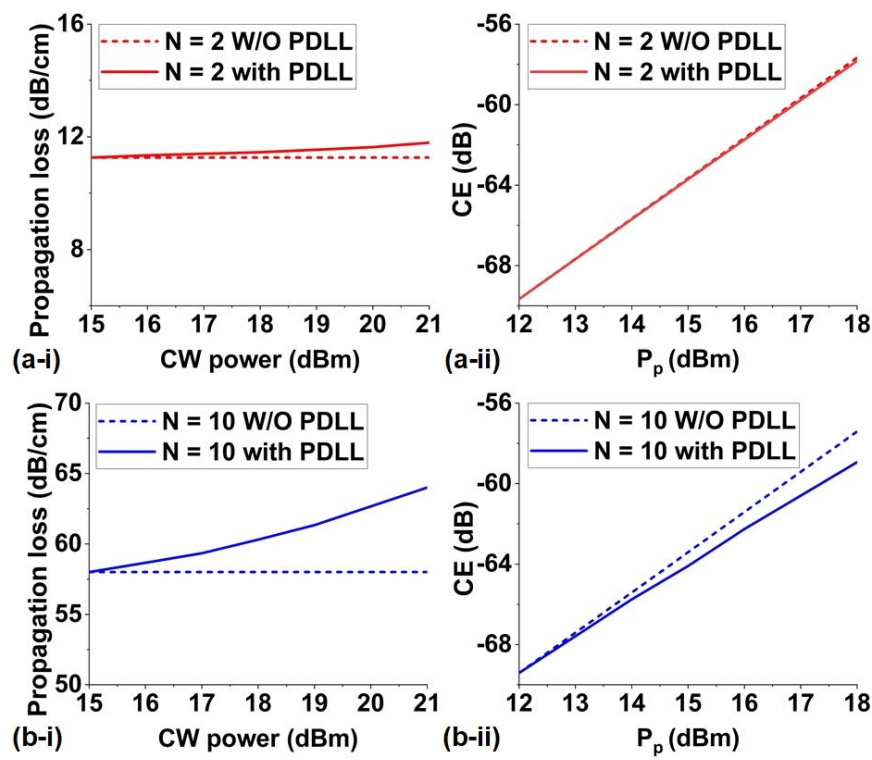

Fig. 7. (a) - (b) Performance comparison of GO-coated SiN waveguides with and without considering GO-induced power dependent linear loss (PDLL) when $N=2$ and $N=10$, respectively. (i) shows propagation loss versus input CW power and (ii) shows CE versus $P_{p}$. In (a) $-(\mathrm{b}), L_{0}=0 \mathrm{~mm}, L=1.5 \mathrm{~mm}$, $W=1600 \mathrm{~nm}$, and $H=660 \mathrm{~nm}$.

\section{DISCUSSION}

In this section, we investigate the influence of the GO coating length, film dispersion, and power dependent loss induced by any photo-thermal changes on the FWM performance, focusing on the $\mathrm{CB}$ and idler power evolution along the hybrid waveguides.

Fig. 7 shows the influence on the $\mathrm{CE}$ of the power-dependent waveguide propagation loss induced by photo-thermal changes in the GO films. Since this has a much slower time response than the nonlinear optical processes of FWM and TPA [34, 46], we accounted for it by adjusting the loss (i.e., $\alpha$ ) of the hybrid waveguides in Eqs. (2) - (4) as a power dependent linear loss (PDLL). In Fig. 7(a), the solid curves show the results for $N=$ 2 when considering the PDLL that slightly increases with input $\mathrm{CW}$ power, whereas the dashed curves represent results that were calculated using the $k$ value of $\mathrm{GO}$ at low powers (i.e., the results for $15 \mathrm{dBm}$ in Fig. 2) without considering the PDLL. The FWM CE slightly decreases when including PDLL, with the difference becoming more significant at higher powers, consistent with the increase of PDLL with CW power. Fig. 7(b) shows the corresponding results when $N=10$, where the difference is more obvious compared to the results for $N=2$, reflecting that there is a more significant influence of the PDLL for the hybrid waveguides with thicker films.

Fig. 8(a) shows the group-velocity dispersion $\beta_{2}$ for the bare $(N=0)$ and coated $\mathrm{SiN}$ waveguides with 2 and 10 layers of GO $(N=2,10)$. As compared with the bare waveguide, the hybrid waveguides display slightly enhanced anomalous dispersion, which becomes more significant as $N$ increases. This could result in better phase matching for FWM and add to the strong Kerr nonlinearity of the GO films.

Fig. 8(b) compares the CB for the bare $(N=0, L=0 \mathrm{~mm})$ versus hybrid waveguides with both uniformly coated $(N=1, L$ $=20 \mathrm{~mm})$ and patterned $(N=1, L=10 \mathrm{~mm}$ and $N=10, L=1$ $\mathrm{mm}$ ) films. The maximum CE is achieved at $N=1, L=10 \mathrm{~mm}$
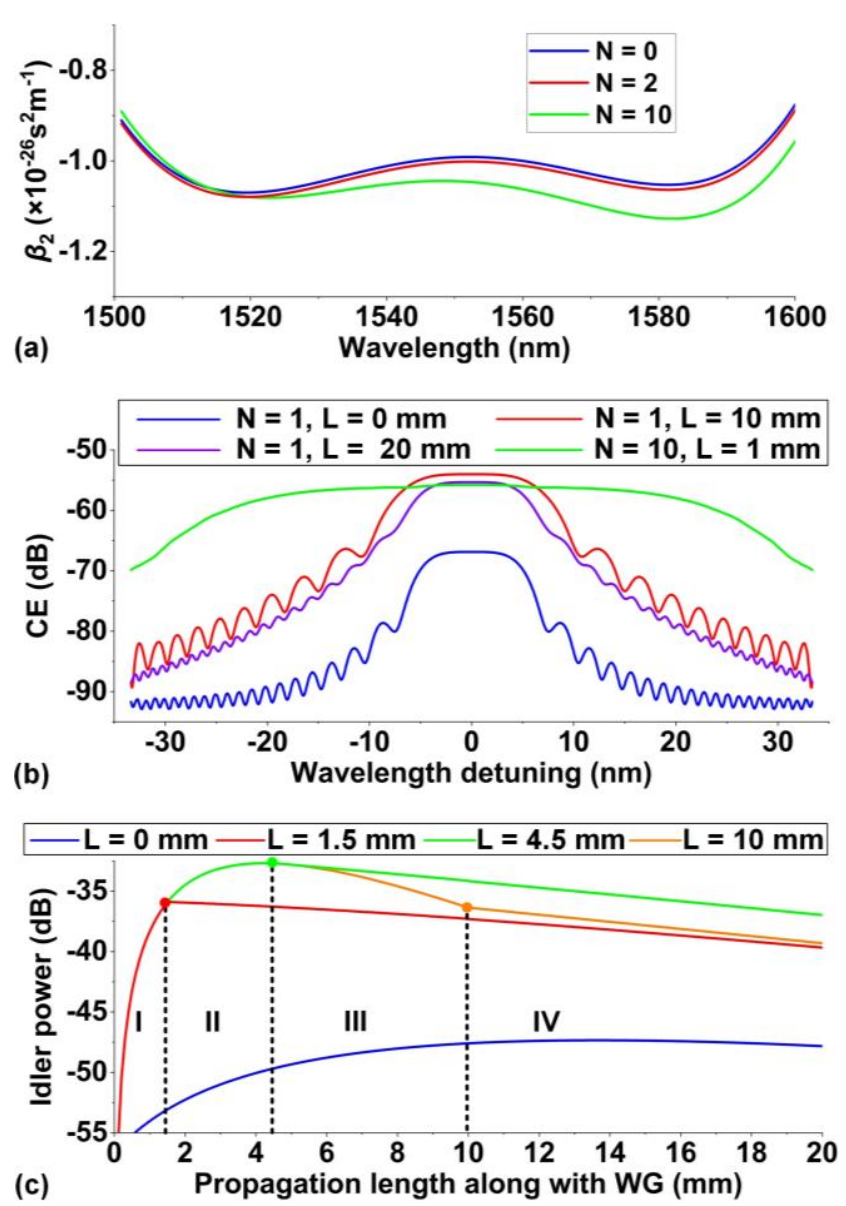

Fig. 8. (a) Group-velocity dispersion for bare and GO-coated SiN waveguides with 2 and 10 layers of GO. (b) CE versus wavelength detuning for bare $\mathrm{SiN}$ waveguides $(N=0, L=0 \mathrm{~mm})$ and hybrid waveguides with both uniformly coated $(N=1, L=20 \mathrm{~mm})$ and patterned $(N=1, L=10 \mathrm{~mm}$ and $N=10, L=1$ $\mathrm{mm})$ GO films. The pump wavelength is fixed at $1550 \mathrm{~nm}$ and the signal wavelength was detuned around $1550 \mathrm{~nm}$. (c) Evolutions of idler power along the hybrid waveguides with different $L$ when $N=2$. In (a) - (c), $L_{0}=0 \mathrm{~mm}, W$ $=1600 \mathrm{~nm}$, and $H=660 \mathrm{~nm}$

due to a better trade-off between $\gamma$ and loss. In theory, the CB can be approximated by [2]:

$$
C B \approx\left[\frac{4 \pi}{\beta_{2} L}\right]^{\frac{1}{2}}
$$

which is inversely proportional to the square root of the product of $\beta_{2}$ and $L$. In Fig. 8(b), the maximum CB is achieved at $N=$ $10, L=1 \mathrm{~mm}$, which is 4.4 times and 4.0 times that of the bare waveguides and the uniformly coated device, respectively. As reflected by Eq. (6), this mainly results from the shorter film length, with a small contribution from the slightly enhanced anomalous dispersion in Fig. 8(a). The broadened CB could also reflect the fact that most of the FWM occurs in the coated segment, where the efficiency is enhanced due to the better phase matching in a shorter coherence length.

Fig. 8(c) shows the evolution of the FWM idler power along the bare $(L=0)$ and hybrid waveguides with different film length $L=1.5 \mathrm{~mm}, 4.5 \mathrm{~mm}$, and $10 \mathrm{~mm}$. The $\mathrm{SiN}$ waveguide was divided into four regions I - IV according to different $L$. It can be seen that the idler power initially rises and then drops after reaching a peak. In the GO coated segment, the idler power reaches a maximum at $L=4.5 \mathrm{~mm}$ due to the trade-off between $\gamma$ and loss, whereas in the uncoated segment the idler power 
decreases continuously because of the propagation loss and relatively low Kerr nonlinearity of the bare SiN waveguides. This further confirms that the enhancement in the Kerr nonlinearity dominates for short film lengths whereas the loss dominates for long GO film lengths. It also provides a criteria for choosing the optimized GO film length in order to maximize the output idler power and hence the CE, and has implications for more advanced photonic integrated circuits [51-53] as well as for applications based on $\mathrm{SiN}$ and Hydex devices for microwave photonics, data communications, neural networks and many other applications [54-83].

\section{CONCLUSION}

In summary, we theoretically optimize FWM in $\mathrm{SiN}$ waveguides integrated with 2D layered GO films. A detailed analysis of the influence of device parameters including waveguide geometry, GO film thickness, length, and position is performed, together with consideration of the influence of GO's dispersion and photo-thermal changes on the FWM performance. By optimizing the device parameters to balance the trade-off between Kerr nonlinearity and loss, up to $\sim 20.7 \mathrm{~dB}$ improvement in FWM efficiency and $~ 4.4$ times increase in FWM bandwidth are achieved. These results highlight the significantly enhanced FWM performance that can be achieved in $\mathrm{SiN}$ waveguides by integrating them with $2 \mathrm{D}$ layered GO films and serve as a roadmap to optimize the overall device performance.

Competing interests: The authors declare no competing interests.

\section{REFERENCES}

[1] R. W. Boyd, Nonlinear optics. London: Elsevier, 2020.

[2] M. A. Foster, A. C. Turner, R. Salem, M. Lipson, and A. L. Gaeta, "Broad-band continuous-wave parametric wavelength conversion in silicon nanowaveguides," Opt. Express, vol. 15, no. 20, pp. 1294912958, Oct. 2007.

[3] D. J. Moss, H. M. v. Driel, and J. E. Sipe, "Third harmonic generation as a structural diagnostic of ion-implanted amorphous and crystalline silicon," Appl. Phys. Lett., vol. 48, no. 17, pp. 1150-1152, Mar. 1986.

[4] M. A. Foster, A. C. Turner, J. E. Sharping, B. S. Schmidt, M. Lipson, and A. L. Gaeta, "Broad-band optical parametric gain on a silicon photonic chip," Nature, vol. 441, no. 7096, pp. 960-963, Jun. 2006.

[5] L. Razzari, D. Duchesne, M. Ferrera, R. Morandotti, S. Chu, B. E. Little, and D. J. Moss, "CMOS-compatible integrated optical hyper-parametric oscillator," Nat. Photonics, vol. 4, no. 1, pp. 41-45, Oct. 2010.

[6] J. S. Levy, A. Gondarenko, M. A. Foster, A. C. Turner-Foster, A. L. Gaeta, and M. Lipson, "CMOS-compatible multiple-wavelength oscillator for on-chip optical interconnects," Nat. Photonics, vol. 4, no. 1, pp. 37-40, Jan. 2010.

[7] B. Corcoran, M. Tan, X. Xu, A. Boes, J. Wu, T. G. Nguyen, S. T. Chu, B. E. Little, R. Morandotti, A. Mitchell and D. J. Moss, "Ultra-dense optical data transmission over standard fibre with a single chip source," Nat. Comm., vol. 11, no. 1, pp. 2568, May 2020.

[8] C. Koos, P. Vorreau, T. Vallaitis, P. Dumon, W. Bogaerts, R. Baets, B. Esembeson, I. Biaggio, T. Michinobu, F. Diederich, W. Freude and J. Leuthold, "All-optical high-speed signal processing with silicon-organic hybrid slot waveguides," Nat. Photonics, vol. 3, no. 4, pp. 216-219, Mar. 2009.

[9] H. Ji, M. Pu, H. Hu, M. Galili, L. K. Oxenløwe, K. Yvind, J. M. Hvam, and P. Jeppesen," "Optical waveform sampling and error-free demultiplexing of $1.28 \mathrm{~Tb} / \mathrm{s}$ serial data in a nanoengineered silicon waveguide," J. Light. Technol., vol. 29, no. 4, pp. 426-431, Feb. 2011.

[10] M. Kues, C. Reimer, P. Roztocki, L. R. Cortés, S. Sciara, B. Wetzel, Y. Zhang, A. Cino, S. T. Chu, B. E. Little, D. J. Moss, L. Caspani, J. Azaña and R. Morandotti, "On-chip generation of high-dimensional entangled quantum states and their coherent control," Nature, vol. 546, no. 7660, pp. 622-626, Jun. 2017.

[11] C. Reimer, M. Kues, P. Roztocki, B. Wetzel, F. Grazioso, B. E. Little, S. T. Chu, T. Johnston, Y. Bromberg, L. Caspani, D. J. Moss, R. Morandotti, "Generation of multiphoton entangled quantum states by means of integrated frequency combs," Science, vol. 351, no. 6278, pp. 1176-1180, Mar. 2016.

[12] M. Kues, C. Reimer, J. M. Lukens, W. J. Munro, A. M. Weiner, D. J. Moss and R. Morandotti, "Quantum optical microcombs," Nat. Photonics, vol. 13, no. 3, pp. 170-179, Mar. 2019.

[13] C. Reimer, S. Sciara, P. Roztocki, M. Islam, L. R. Cortés, Y. Zhang, B. Fischer, S. Loranger, R. Kashyap, A. Cino, S. T. Chu, B. E. Little, D. J. Moss, L. Caspani, W. J. Munro, J. Azaña, M. Kues and R. Morandotti, "High-dimensional one-way quantum processing implemented on dlevel cluster states," Nat. Phys., vol. 15, no. 2, pp. 148-153, Feb. 2019.

[14] A. Pasquazi, M. Peccianti, Y. Park, B. E. Little, S. T. Chu, R. Morandotti, J. Azaña and D. J. Moss, "Sub-picosecond phase-sensitive optical pulse characterization on a chip," Nat. Photonics, vol. 5, no. 10, pp. 618-623, Aug. 2011.

[15] M. A. Foster, R. Salem, D. F. Geraghty, A. C. Turner-Foster, M. Lipson, and A. L. Gaeta, "Silicon-chip-based ultrafast optical oscilloscope," Nature, vol. 456, no. 7218, pp. 81-4, Nov. 2008.

[16] H. Bao, A. Cooper, M. Rowley, L. Di Lauro, J. Sebastian, T. Gongora, S. T. Chu, B. E. Little, G. Oppo, R. Morandotti, D. J. Moss, B. Wetzel, M. Peccianti and A. Pasquazi, "Laser cavity-soliton microcombs," Nat. Photonics, vol. 13, no. 6, pp. 384-389, Jun. 2019.

[17] M.Kues, et. al., "Passively modelocked laser with an ultra-narrow spectral width", Nat. Photonics, vol. 11, no. 3, 159 (2017). DOI:10.1038/nphoton.2016.271

[18] R. Salem, M. A. Foster, A. C. Turner, D. F. Geraghty, M. Lipson, and A. L. Gaeta, "Signal regeneration using low-power four-wave mixing on silicon chip," Nat. Photonics, vol. 2, no. 1, pp. 35-38, Jan. 2008.

[19] D. J. Moss, R. Morandotti, A. L. Gaeta, and M. Lipson, "New CMOScompatible platforms based on silicon nitride and Hydex for nonlinear optics," Nat. Photonics, vol. 7, no. 8, pp. 597-607, Jul. 2013.

[20] J. Leuthold, C. Koos, and W. Freude, "Nonlinear silicon photonics," Nat. Photonics, vol. 4, no. 8, pp. 535-544, Jul. 2010.

[21] T. Jiang, V. Kravtsov, M. Tokman, A. Belyanin, and M. B. Raschke, "Ultrafast coherent nonlinear nanooptics and nanoimaging of graphene," Nat. Nanotechnol., vol. 14, no. 9, pp. 838-843, Aug. 2019.

[22] M. Li, L. Zhang, L. Tong, and D. Dai, "Hybrid silicon nonlinear photonics [Invited]," Photonics Res., vol. 6, no. 5, pp. B13-B22, May 2018.

[23] J. Wu, X. Xu, T. G. Nguyen, S. T. Chu, B. E. Little, R. Morandotti, "RF photonics: an optical microcombs' perspective," IEEE J. Sel. Topics Quantum Electron., vol. 24, no. 4, pp. 1-20, Feb. 2018.

[24] T. Gu, N. Petrone, J. F. McMillan, A. van der Zande, M. Yu, G. Q. Lo, D. L. Kwong, J. Hone and C. W. Wong, "Regenerative oscillation and four-wave mixing in graphene optoelectronics," Nat. Photonics, vol. 6, no. 8, pp. 554-559, Aug. 2012.

[25] K. Alexander, N. A. Savostianova, S. A. Mikhailov, B. Kuyken, and D. Van Thourhout, "Electrically tunable optical nonlinearities in graphenecovered SiN waveguides characterized by four-wave mixing," ACS Photonics, vol. 4, no. 12, pp. 3039-3044, Aug. 2017.

[26] Y. Yang, J. Wu, X. Xu, Y. Liang, S. T. Chu, B. E. Little, R. Morandotti, B. Jia, and D. J. Moss, "Invited Article: Enhanced four-wave mixing in waveguides integrated with graphene oxide," APL Photonics, vol. 3, no. 12, pp. 120803, Oct. 2018.

[27] J. Zheng, Z. Yang, C. Si, Z. Liang, X. Chen, R. Cao, Z. Guo, K. Wang, Y. Zhang, J. Ji, M. Zhang, D. Fan, and H. Zhang, "Black phosphorus based all-optical-signal-processing: toward high performances and enhanced stability," ACS Photonics, vol. 4, no. 6, pp. 1466-1476, May 2017.

[28] M. Ji, H. Cai, L. Deng, Y. Huang, Q. Huang, J. Xia, Z. Li, J. Yu, and Y. Wang, "Enhanced parametric frequency conversion in a compact silicongraphene microring resonator," Opt. Express, vol. 23, no. 14, pp. 1867985, Jul. 2015.

[29] K. P. Loh, Q. Bao, G. Eda, and M. Chhowalla, "Graphene oxide as a chemically tunable platform for optical applications," Nat. Chem., vol. 2, no. 12, pp. 1015-24, Dec. 2010.

[30] O. C. Compton and S. T. Nguyen, "Graphene oxide, highly reduced graphene oxide, and graphene: versatile building blocks for carbon-based materials," Small, vol. 6, no. 6, pp. 711-23, Mar. 2010.

[31] X. Zheng, B. Jia, X. Chen, and M. Gu, "In situ third-order non-linear responses during laser reduction of graphene oxide thin films towards 
on-chip non-linear photonic devices," Adv. Mater., vol. 26, no. 17, pp. 2699-703, May 2014.

[32] X. Xu, X. Zheng, F. He, Z. Wang, H. Subbaraman, Y. Wang, B. Jia and R. T. Chen, "Observation of third-order nonlinearities in graphene oxide film at telecommunication wavelengths," Sci. Rep., vol. 7, no. 1, pp. 9646, Aug. 2017.

[33] W. Gao, "The chemistry of graphene oxide," in Graphene Oxide: Reduction Recipes, Spectroscopy, and Applications, W. Gao, Ed. Cham: Springer International Publishing, 2015, pp. 61-95.

[34] Y. Qu, J. Wu, Y. Yang, Y. Zhang, Y. Liang, H. El Dirani, R. Crochemore, P. Demongodinc, C. Sciancalepore, C. Grillet, C. Monat, B. Jia, D. J. Moss, "Enhanced nonlinear four-wave mixing in silicon nitride waveguides integrated with 2D layered graphene oxide films", Adv. Opt. Mater., vol. 8, no. 20, pp. 2001048 Oct. 2020. DOI: $10.1002 /$ adom.202001048.

[35] Y. Yang, H. Lin, B. Zhang, Y. Zhang, X. Zheng, A. Yu, M. Hong, and B. Jia, "Graphene-based multilayered metamaterials with phototunable architecture for on-chip photonic devices," ACS Photonics, vol. 6, no. 4, pp. 1033-1040, Feb. 2019

[36] J. Wu, Y. Yang, Y. Qu, X. Xu, Y. Liang, S. T. Chu, B. E. Little, R. Morandotti, B. Jia, and D. J. Moss, "Graphene oxide waveguide and micro-ring resonator polarizers," Laser Photonics Rev., vol. 13, no. 9, 1900056, Sep. 2019.

[37] H. Lin, Y. Song, Y. Huang, D. Kita, S. Deckoff-Jones, K. Wang, L. Li, J. Li, H. Zheng, Z. Luo, H. Wang, S. Novak, A. Yadav, C. Huang, R. Shiue, D. Englund, T. Gu, D. Hewak, K. Richardson, J. Kong and J. Hu, "Chalcogenide glass-on-graphene photonics," Nat. Photonics, vol. 11, no. 12, pp. 798-805, Oct. 2017.

[38] F. Bonaccorso, Z. Sun, T. Hasan, and A. C. Ferrari, "Graphene photonics and optoelectronics," Nat. Photonics, vol. 4, no. 9, pp. 611-622, Sep. 2010.

[39] H. El Dirani, A. Kamel, M. Casale, S. Kerdiles, C. Monat, X. Letartre, M. Pu, L. K. Oxenløwe, K. Yvind, and C. Sciancalepore, "Annealingfree $\mathrm{Si}_{3} \mathrm{~N}_{4}$ frequency combs for monolithic integration with $\mathrm{Si}$ photonics," Appl. Phys. Lett., vol. 113, no. 8, pp. 081102, Aug. 2018.

[40] P. Demongodin, H. El Dirani, J. Lhuillier, R. Crochemore, M. Kemiche, T. Wood, S. Callard, P. Rojo-Romeo, C. Sciancalepore, C. Grillet, and C. Monat, "Ultrafast saturable absorption dynamics in hybrid graphene/ $\mathrm{Si}_{3} \mathrm{~N}_{4}$ waveguides," Apl Photonics, vol. 4, no. 7, Jul. 2019.

[41] L. Liu, K. Xu, X. Wan, J. Xu, C. Y. Wong, and H. K. Tsang, "Enhanced optical Kerr nonlinearity of $\mathrm{MoS}_{2}$ on silicon waveguides," Photonics Res., vol. 3, no. 5, pp. 206, Aug. 2015.

[42] Y. Yang, R. Liu, J. Wu, X. Jiang, P. Cao, X. Hu, T. Pan, C. Qiu, J. Yang, Y. Song, D. Wu and Y. Su, "Bottom-up fabrication of graphene on silicon/silica substrate via a facile soft-hard template approach," Sci. Rep., vol. 5, pp. 13480, Aug. 2015.

[43] H. Li, Y. Anugrah, S. J. Koester, and M. Li, "Optical absorption in graphene integrated on silicon waveguides," Appl. Phys. Lett., vol. 101, no. 11, pp. 111110, Sep. 2012.

[44] H. Cai, Y. Cheng, H. Zhang, Q. Huang, J. Xia, R. Barille, and Y. Wang, "Enhanced linear absorption coefficient of in-plane monolayer graphene on a silicon microring resonator," Opt. Express, vol. 24, no. 21, pp. 24105-24116, Oct. 2016.

[45] W. Y. Chong, W. H. Lim, Y. K. Yap, C. K. Lai, R. M. De La Rue, and H. Ahmad, "Photo-induced reduction of graphene oxide coating on optical waveguide and consequent optical intermodulation," Sci. Rep., vol. 6, no. 1, pp. 23813, Apr. 2016.

[46] J. Wu, Y. Yang, Y. Qu, L. Jia, Y. Zhang, X. Xu, S. T. Chu, B. E. Little, R. Morandotti, B. Jia, and D. J. Moss, "2D layered graphene oxide films integrated with micro-ring resonators for enhanced nonlinear optics," Small, pp. e1906563, Mar. 112020.

[47] Y. Zhang, J. Wu, Y. Yang, Y. Qu, L. Jia, T. Moein, B. Jia, and D. J. Moss, "Enhanced kerr nonlinearity and nonlinear figure of merit in silicon nanowires integrated with 2D graphene oxide films," ACS Applied Materials \& Interfaces, vol. 12, no. 29, pp. 33094-33103, Jul. 2020.

[48] D. J. Moss, H. M. van Driel, and J. E. Sipe, "Dispersion in the anisotropy of optical third-harmonic generation in silicon," Opt. Lett., vol. 14, no. 1, pp. 57-59, Jan. 1989.

[49] Q. Lin, J. Zhang, P. M. Fauchet, and G. P. Agrawal, "Ultrabroadband parametric generation and wavelength conversion in silicon waveguides," Opt. Express, vol. 14, no. 11, pp. 4786-4799, May 2006.

[50] Q. A. Liu, S. M. Gao, Z. Q. Li, Y. Q. Xie, and S. L. He, "Dispersion engineering of a silicon-nanocrystal-based slot waveguide for broadband wavelength conversion," Appl. Opt., vol. 50, no. 9, pp. 1260-1265, Mar. 2011.

[51] J. Wu, T. Moein, X. Xu, and D. J. Moss, "Advanced photonic filters based on cascaded Sagnac loop reflector resonators in silicon-oninsulator nanowires," APL Photonics, vol. 3, 046102 (2018). DOI:/10.1063/1.5025833Apr. 2018.

[52] J. Wu, T. Moein, X. Xu, G. H. Ren, A. Mitchell, and D. J. Moss, "Microring resonator quality factor enhancement via an integrated Fabry-Perot cavity," APL Photonics, vol. 2, 056103 (2017).

[53] J. Wu, L. Jia, Y. Zhang, Y. Qu, B. Jia, and D. J. Moss, "Graphene oxide for integrated photonics and flat optics," Adv. Mater., vol. 33, no. 3, pp. 200415, Jan. 2021.

[54]. X. Xu, et al., "Broadband RF channelizer based on an integrated optical frequency Kerr comb source," Journal of Lightwave Technology, vol. 36, no. 19, pp. 4519-4526 (2018).

[55]. X. Xu, et al., "Continuously tunable orthogonally polarized RF optical single sideband generator based on micro-ring resonators," Journal of Optics, vol. 20, no. 11, 115701 (2018).

[56]. X. Xu, et al., "Orthogonally polarized RF optical single sideband generation and dual-channel equalization based on an integrated microring resonator," Journal of Lightwave Technology, vol. 36, no. 20, pp. 4808-4818 (2018).

[57].X. Xu, et al., "Photonic microwave true time delays for phased array antennas using a $49 \mathrm{GHz}$ FSR integrated optical micro-comb source," Photonics Res, vol. 6, no. 5, pp. B30-B36 (2018).

[58]. X. Xu, et al., "Advanced adaptive photonic RF filters with 80 taps based on an integrated optical micro-comb source," Journal of Lightwave Technology, vol. 37, no. 4, pp. 1288-1295 (2019).

[59]. X. Xu et al., Broadband microwave frequency conversion based on an integrated optical micro-comb source", Journal of Lightwave Technology, vol. 38 no. 2, pp. 332-338 (2020).

[60].M. Tan et al., "Photonic RF and microwave filters based on $49 \mathrm{GHz}$ and 200GHz Kerr microcombs", Optics Comm. vol. 465, Article: 125563, Feb. $22(2020)$.

[61]. X. Xu et al., "Broadband photonic RF channelizer with 90 channels based on a soliton crystal microcomb", Journal of Lightwave Technology, Vol. 38, no. 18, pp.5116 - 5121 (2020). doi: 10.1109/JLT.2020.2997699.

[62]. X. Xu et al., "Photonic RF and microwave integrator with soliton crystal microcombs", IEEE Transactions on Circuits and Systems II: Express Briefs, Vol. 67 (12) 3582-3586 (2020). DOI:10.1109/TCSII.2020.2995682.

[63].X. Xu et al., "Photonic RF phase-encoded signal generation with a microcomb source", Journal of Lightwave Technology, vol. 38, no. 7, pp. $1722-1727$ (2020).

[64]. X. Xu et al., "High performance RF filters via bandwidth scaling with Kerr micro-combs," APL Photonics, vol. 4, no. 2, pp. 026102 (2019).

[65]. M. Tan et al., "Microwave and RF photonic fractional Hilbert transformer based on a $50 \mathrm{GHz}$ Kerr micro-comb", Journal of Lightwave Technology, vol. 37, no. 24, pp. 6097 - 6104 (2019).

[66].M. Tan et al., "RF and microwave fractional differentiator based on photonics", IEEE Transactions on Circuits and Systems: Express Briefs, Vol. 67, No. 11, pp. 2767 - 2771 (2020). DOI:10.1109/TCSII.2020.2965158.

[67]. M. Tan et al., "Photonic RF arbitrary waveform generator based on a soliton crystal micro-comb source", Journal of Lightwave Technology, Vol. 38, No. 22, pp. 6221-6226, Oct 22 (2020). DOI: 10.1109/JLT.2020.3009655.

[68].M. Tan, X. Xu, J. Wu, R. Morandotti, A. Mitchell, and D. J. Moss, "RF and microwave high bandwidth signal processing based on Kerr Micro-combs", Advances in Physics X, VOL. 6, NO. 1, 1838946 (2020). DOI:10.1080/23746149.2020.1838946.

[69]. Mengxi Tan, Xingyuan Xu, Jiayang Wu, Thach G. Nguyen, Sai T. Chu, Brent E. Little, Roberto Morandotti, Arnan Mitchell, and David J. Moss, "Photonic Radio Frequency Channelizers based on Kerr Micro-combs and Integrated Micro-ring Resonators", JOSarXiv.202010.0002.

[70]. Mengxi Tan, Xingyuan Xu, David Moss "Tunable Broadband RF Photonic Fractional Hilbert Transformer Based on a Soliton Crystal Microcomb", Preprints, DOI: 10.20944/preprints202104.0162.v1

[71]. Mengxi Tan, X. Xu, J. Wu, T. G. Nguyen, S. T. Chu, B. E. Little, R. Morandotti, A. Mitchell, and David J. Moss, "Orthogonally polarized Photonic Radio Frequency single sideband generation with integrated micro-ring resonators", Journal of Semiconductors $\underline{\mathbf{4 2}}$ (4), 041305 (2021). DOI: 10.1088/1674-4926/42/4/041305.

[72]. Mengxi Tan, X. Xu, J. Wu, T. G. Nguyen, S. T. Chu, B. E. Little, R. Morandotti, A. Mitchell, and David J. Moss, "Photonic Radio Frequency Channelizers based on Kerr Optical Micro-combs", Journal of Semiconductors $\underline{42}$ (4), 041302 (2021). (ISSN 1674-4926). 


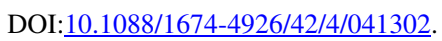

[73]. H.Bao, L.Olivieri, M.Rowley, S.T. Chu, B.E. Little, R.Morandotti, D.J. Moss, J.S.T. Gongora, M.Peccianti and A.Pasquazi, "Laser Cavity Solitons and Turing Patterns in Microresonator Filtered Lasers: properties and perspectives", Paper No. LA203-5, Paper No. 11672-5, SPIE LASE, SPIE Photonics West, San Francisco CA March 6-11 (2021). DOI: $10.1117 / 12.2576645$

[74]. Mengxi Tan, X. Xu, J. Wu, A. Boes, T. G. Nguyen, S. T. Chu, B. E. Little, R. Morandotti, A. Mitchell, and David J. Moss, "Advanced microwave signal generation and processing with soliton crystal microcombs", or "Photonic convolutional accelerator and neural network in the Tera-OPs regime based on Kerr microcombs", Paper No. 11689-38, PW21OOE201-67, Integrated Optics: Devices, Materials, and Technologies XXV, SPIE Photonics West, San Francisco CA March 6-11 (2021). DOI: $\underline{10.1117 / 12.2584017}$

[75]. Mengxi Tan, Bill Corcoran, Xingyuan Xu, Andrew Boes, Jiayang Wu, Thach Nguyen, Sai T. Chu, Brent E. Little, Roberto Morandotti, Arnan Mitchell, and David J. Moss, "Optical data transmission at 40 Terabits/s with a Kerr soliton crystal microcomb", Paper No.11713-8, PW21OOE803-23, Next-Generation Optical Communication: Components, Sub-Systems, and Systems X, SPIE Photonics West, San Francisco CA March 6-11 (2021). DOI:10.1117/12.2584014

[76]. Mengxi Tan, X. Xu, J. Wu, A. Boes, T. G. Nguyen, S. T. Chu, B. E. Little, R. Morandotti, A. Mitchell, and David J. Moss, "RF and microwave photonic, fractional differentiation, integration, and Hilbert transforms based on Kerr micro-combs", Paper No. 11713-16, PW21O-OE803-24, Next-Generation Optical Communication: Components, Sub-Systems, and Systems X, SPIE Photonics West, San Francisco CA March 6-11 (2021). DOI:10.1117/12.2584018

[77]. Mengxi Tan, X. Xu, J. Wu, A. Boes, T. G. Nguyen, S. T. Chu, B. E. Little, R. Morandotti, A. Mitchell, and David J. Moss, "Broadband photonic RF channelizer with 90 channels based on a soliton crystal microcomb", or "Photonic microwave and RF channelizers based on Kerr micro-combs", Paper No. 11685-22, PW21O-OE106-49, Terahertz, RF, Millimeter, and Submillimeter-Wave Technology and Applications XIV, SPIE Photonics West, San Francisco CA March 6-11 (2021). DOI: $10.1117 / 12.2584015$

[78]. X. Xu, M. Tan, J. Wu, S. T. Chu, B. E. Little, R. Morandotti, A. Mitchell, B. Corcoran, D. Hicks, and D. J. Moss, "Photonic perceptron based on a Kerr microcomb for scalable high speed optical neural networks", IEEE Topical Meeting on Microwave Photonics (MPW), pp. 220224,.Matsue, Japan, November 24-26, 2020. Electronic ISBN:978-488552-331-1.

DOI: $\underline{\text { 10.23919/MWP48676.2020.9314409 }}$

[79]. Mengxi Tan, Bill Corcoran, Xingyuan Xu, Andrew Boes, Jiayang Wu, Thach Nguyen, S.T. Chu, B. E. Little, Roberto Morandotti, Arnan Mitchell, and David J. Moss, "Ultra-high bandwidth optical data transmission with a microcomb", IEEE Topical Meeting on Microwave Photonics (MPW), pp. 78-82.Virtual Conf., Matsue, Japan, November 24-26, 2020. Electronic ISBN:978-4-88552-331-1.

DOI: $10.23919 / M W P 48676.2020 .9314476$

[80]. T. G. Nguyen et al., "Integrated frequency comb source-based Hilbert transformer for wideband microwave photonic phase analysis," Opt. Express, vol. 23, no. 17, pp. 22087-22097 (2015).

[81]. X. Xu, J. Wu, M. Shoeiby, T. G. Nguyen, S. T. Chu, B. E. Little, R. Morandotti, A. Mitchell, and D. J. Moss, "Reconfigurable broadband microwave photonic intensity differentiator based on an integrated optical frequency comb source," APL Photonics, vol. 2, no. 9, 096104 (2017).

[82]. X. Xu, M. Tan, J. Wu, R. Morandotti, A. Mitchell, and D. J. Moss, "Microcomb-based photonic RF signal processing", IEEE Photonics Technology Letters, vol. 31 no. 23 1854-1857 (2019).

[83]. X. Xu, et al., "Advanced RF and microwave functions based on an integrated optical frequency comb source," Opt. Express, vol. 26, no. 3, pp. 2569-2583 (2018). 


\section{Figures}

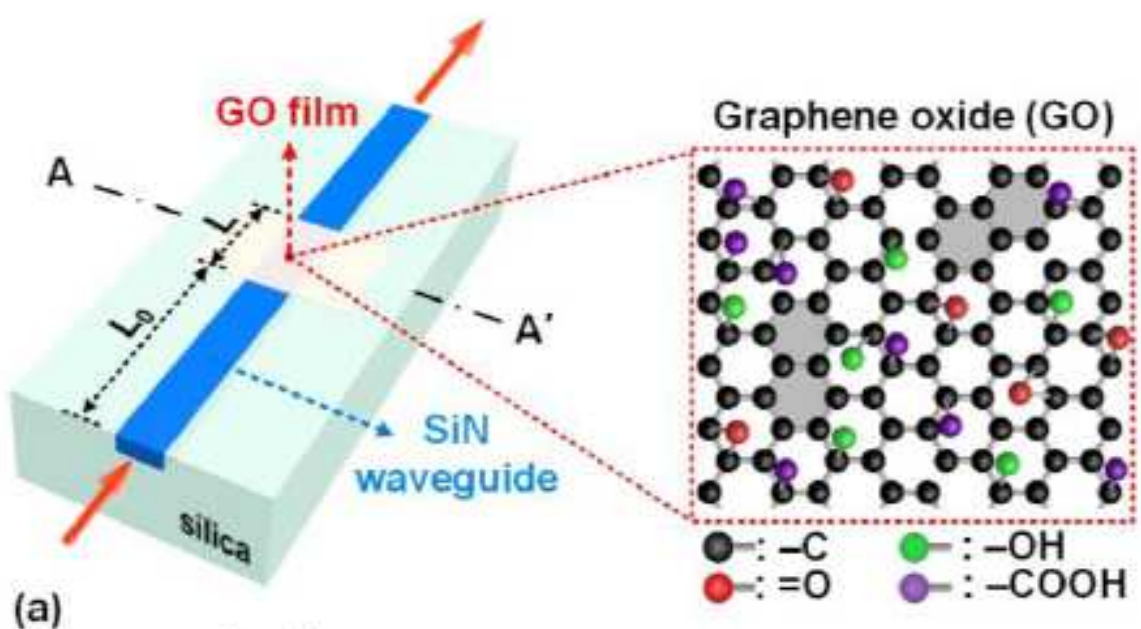

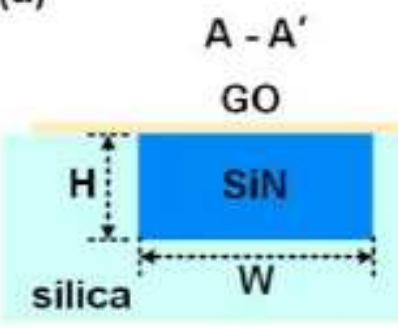

(b)

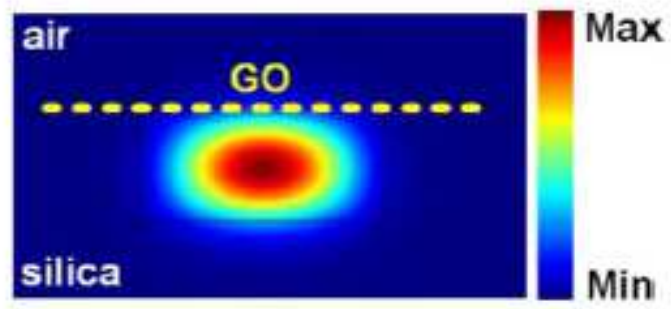

(c)

\section{Figure 1}

(a) Schematic illustration of a SiN waveguide coated with 10 layers of GO. Inset shows schematics of atomic structure of GO. (b) Schematic illustration of the cross section of the hybrid waveguide in (a). (c) TE mode profile corresponding to (b). The definitions of $L O, L, W$, and $\mathrm{H}$ are given in Table I. 

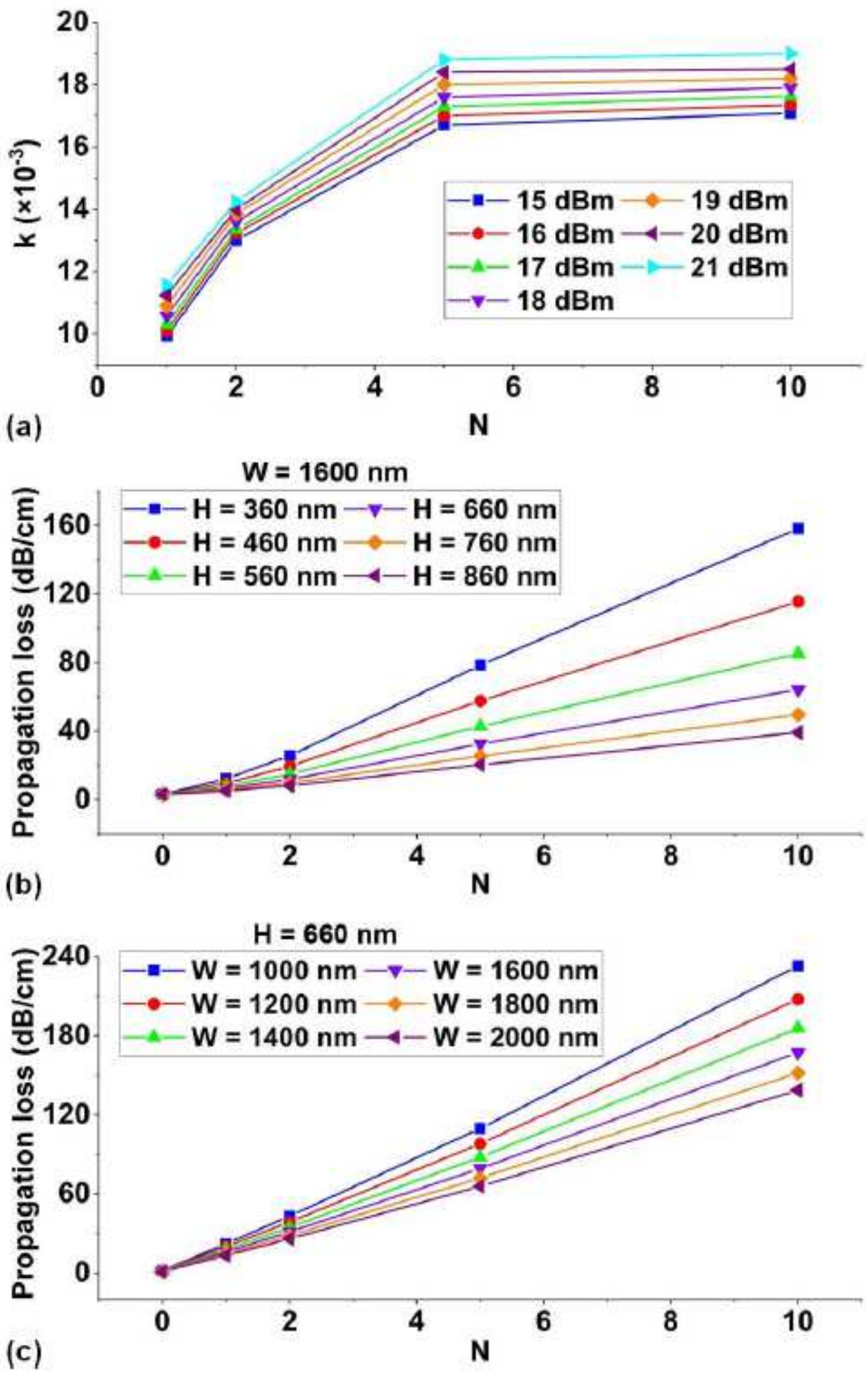

Figure 2

(a) Extinction coefficient $\mathrm{k}$ of $\mathrm{GO}$ versus $\mathrm{N}$ under various input $\mathrm{CW}$ powers. (b) Propagation loss versus $\mathrm{N}$ for GO-coated SiN waveguides with various $\mathrm{H}$ when $\mathrm{W}=1600 \mathrm{~nm}$. (c) Propagation loss versus $\mathrm{N}$ for GOcoated $\mathrm{SiN}$ waveguides with various $\mathrm{W}$ when $\mathrm{H}=660 \mathrm{~nm}$. The points at $\mathrm{N}=0$ correspond to the results for bare $\mathrm{SiN}$ waveguides. 

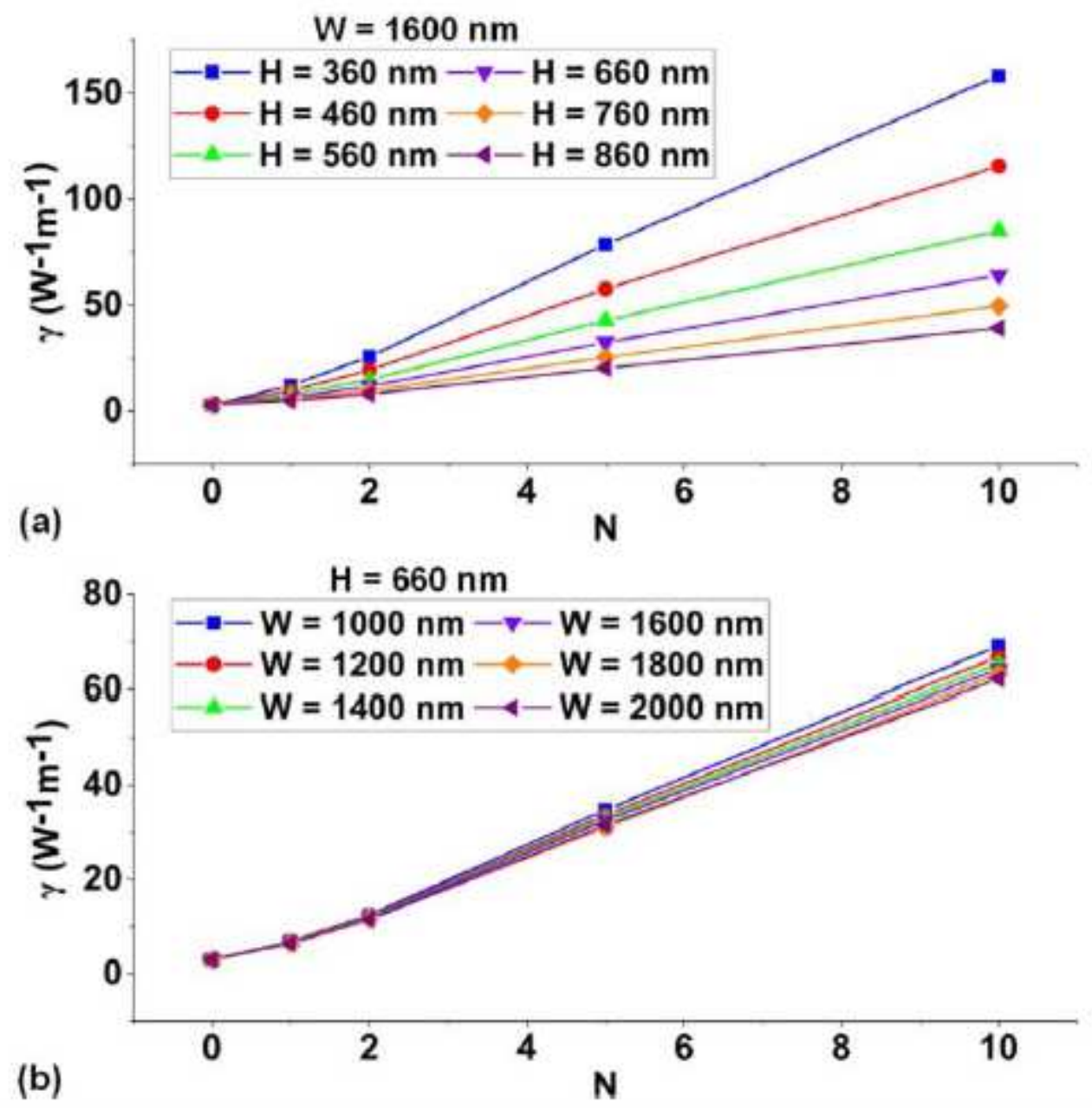

Figure 3

Nonlinear parameter $\mathrm{Y}$ versus $\mathrm{N}$ for GO-coated SiN waveguides with (a) various $\mathrm{H}$ when $\mathrm{W}=1600 \mathrm{~nm}$ (b) various $\mathrm{W}$ when $\mathrm{H}=660 \mathrm{~nm}$. The points at $\mathrm{N}=0$ correspond to the results for bare SiN waveguides. 

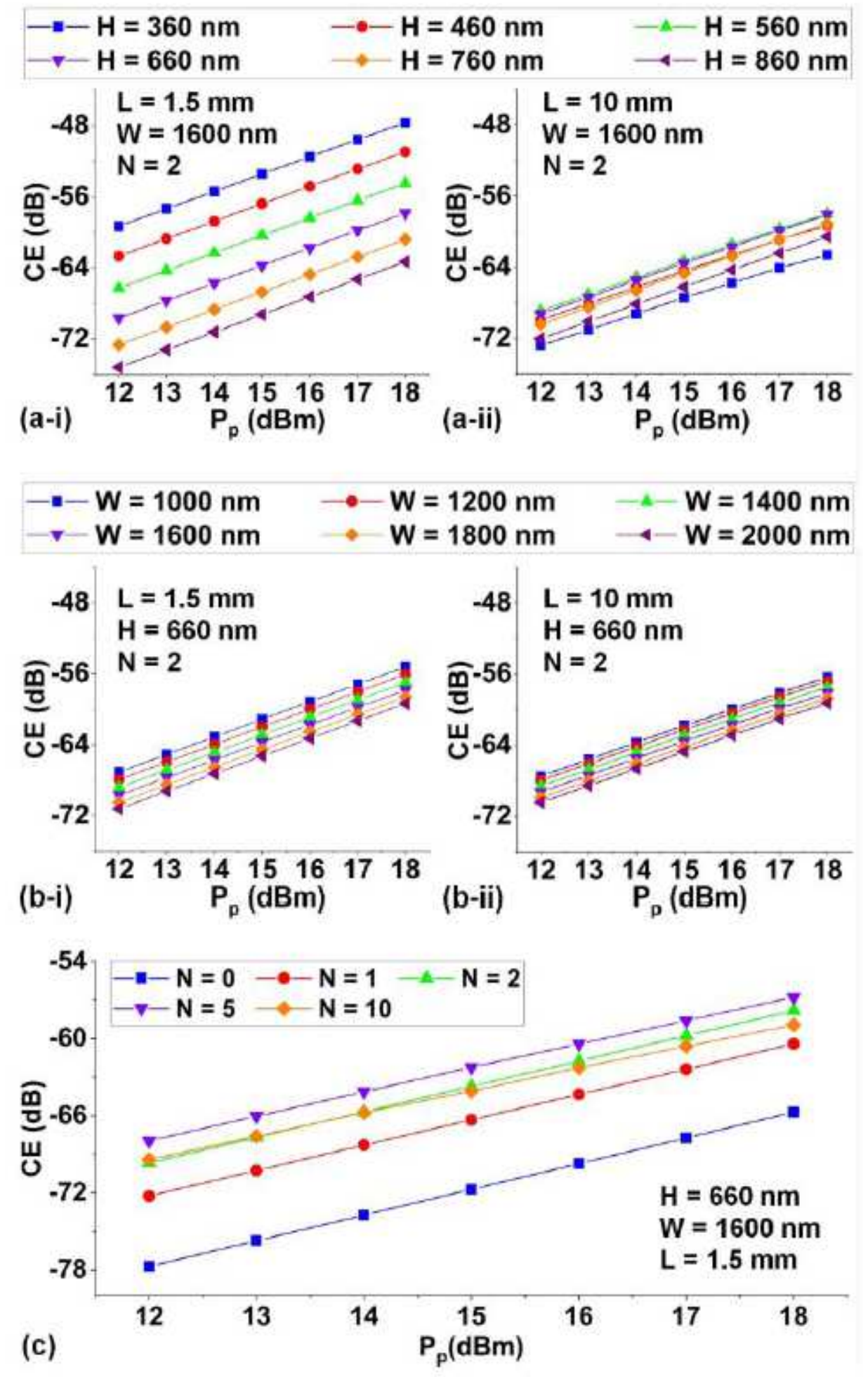

Figure 4

FWM CE versus Pp for GO-coated SiN waveguides with various (a) $\mathrm{H}$, (b) W, and (c) N. In (a) and (b), N = 2, (i) and (ii) show the results for $L=1.5 \mathrm{~mm}$ and $L=10 \mathrm{~mm}$, respectively. In (a) and (c), $W=1600 \mathrm{~nm}$. In (b) and (c), H = $660 \mathrm{~nm}$. In (a) - (c), LO = 0. 

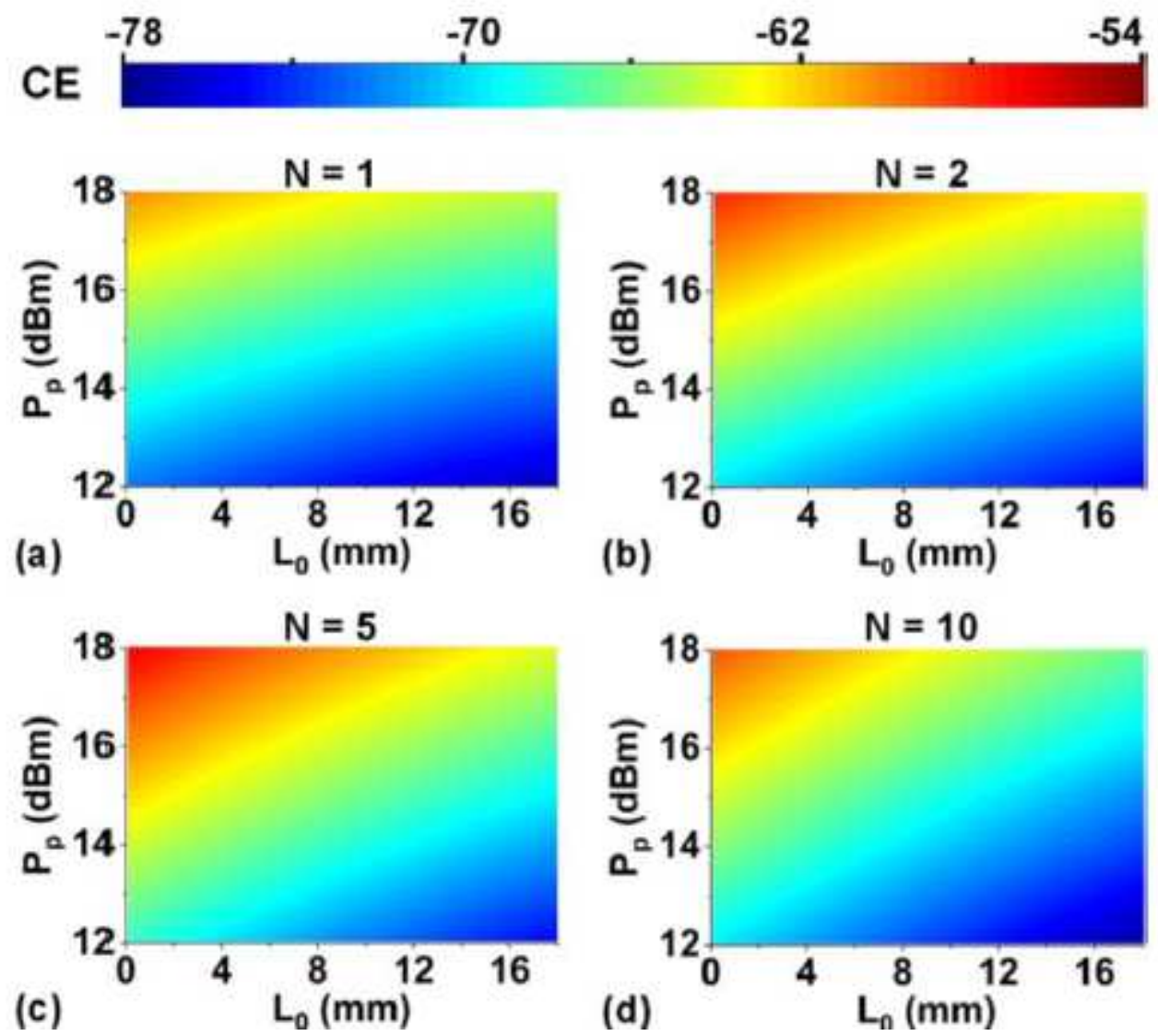

Figure 5

FWM CE of GO-coated SiN waveguides versus $L 0$ and Pp when (a) $N=1$, (b) $N=2$, (c) $N=5$, and (d) $N=$ 10. $\ln (\mathrm{a})-(\mathrm{d}), \mathrm{L}=1.5 \mathrm{~mm}, \mathrm{H}=660 \mathrm{~nm}$, and $\mathrm{W}=1600 \mathrm{~nm}$.
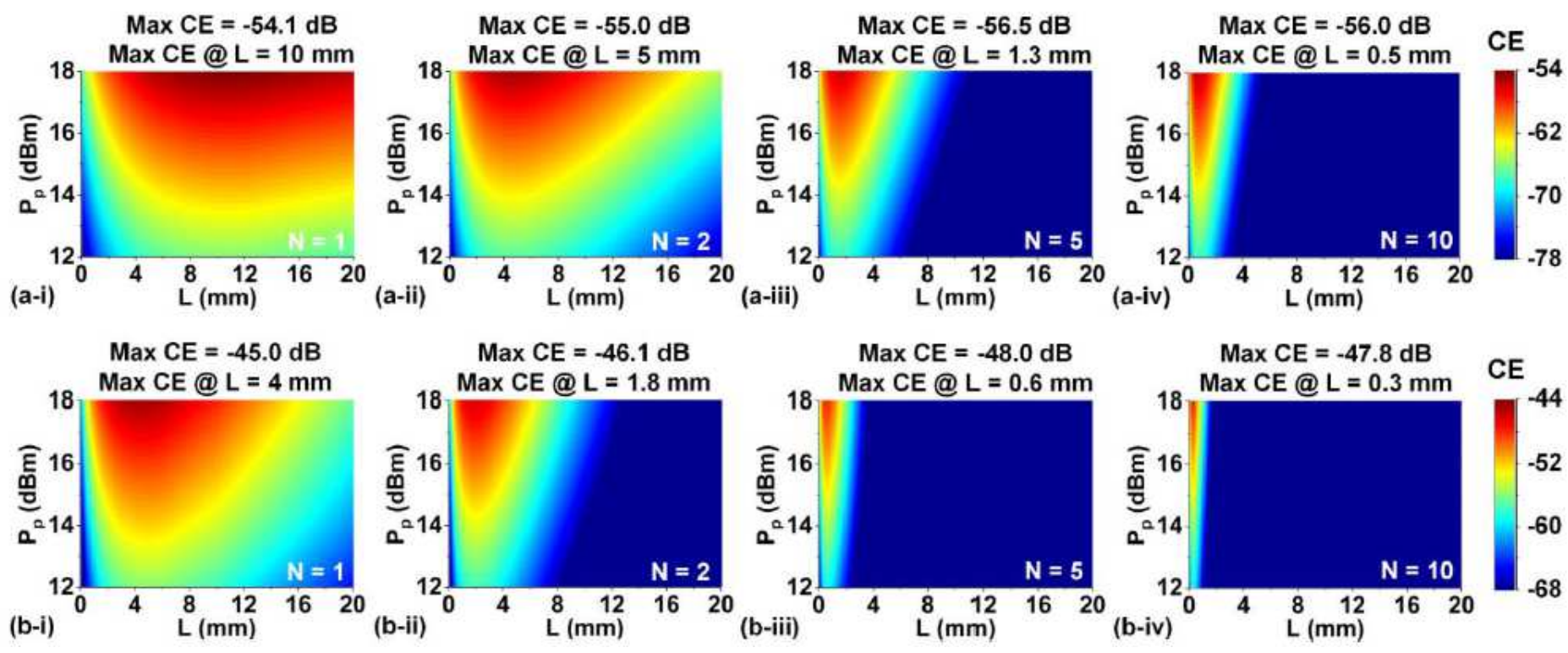

Figure 6 
FWM CE of GO-coated SiN waveguides versus $L$ and Pp when (a) W $=1600 \mathrm{~nm}, \mathrm{H}=660 \mathrm{~nm}$ and (b) W = $1000 \mathrm{~nm}, \mathrm{H}=360 \mathrm{~nm}$. (i) - (iv) show the results for $\mathrm{N}=1,2,5$, and 10, respectively. In (a) and (b), L0 = 0 $\mathrm{mm}$.
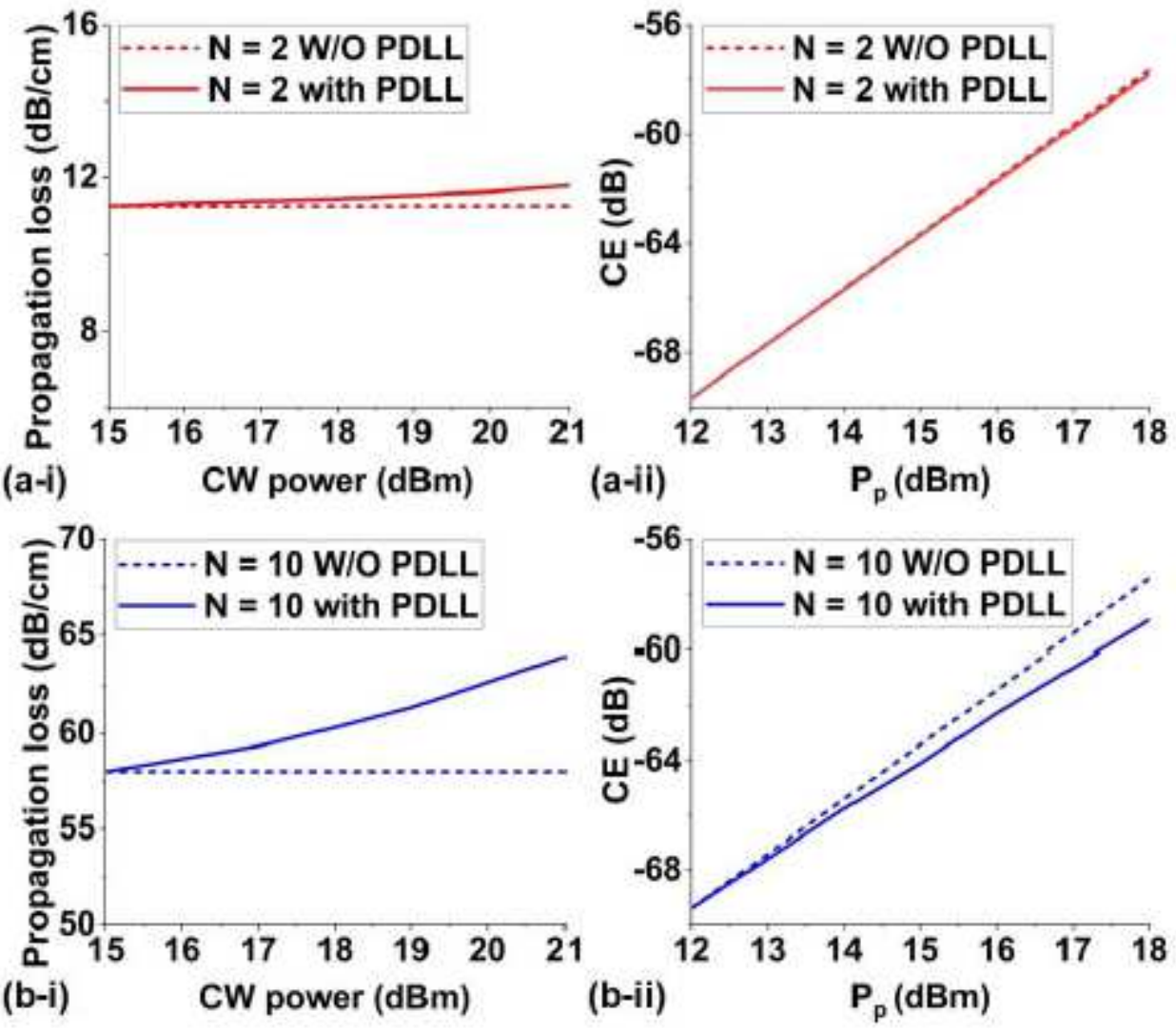

Figure 7

(a) - (b) Performance comparison of GO-coated SiN waveguides with and without considering GOinduced power dependent linear loss (PDLL) when $N=2$ and $N=10$, respectively. (i) shows propagation loss versus input $C W$ power and (ii) shows CE versus Pp. In (a) - (b), $L 0=0 \mathrm{~mm}, \mathrm{~L}=1.5 \mathrm{~mm}, \mathrm{~W}=1600$ $\mathrm{nm}$, and $\mathrm{H}=660 \mathrm{~nm}$. 

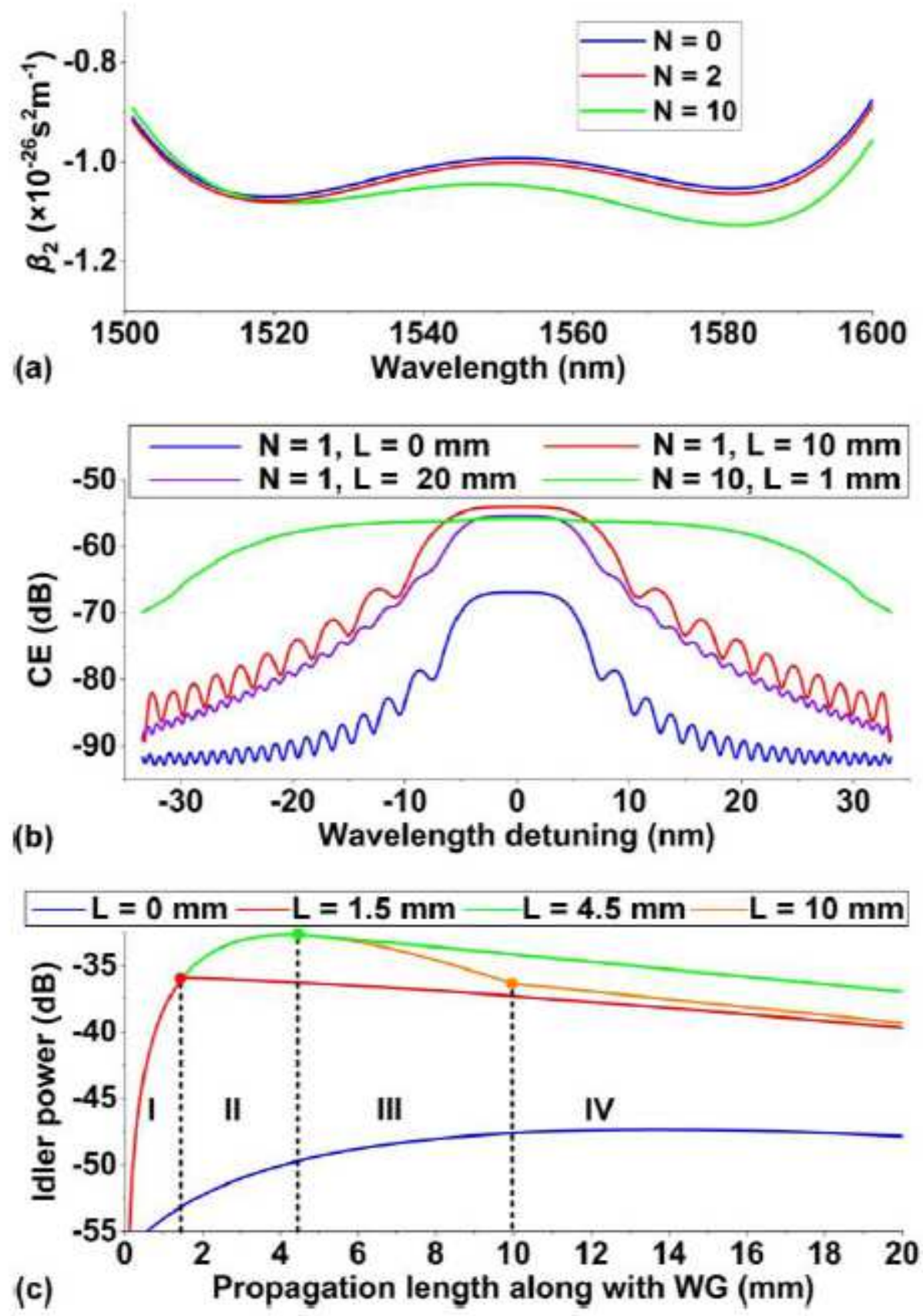

Figure 8

(a) Group-velocity dispersion for bare and GO-coated SiN waveguides with 2 and 10 layers of GO. (b) CE versus wavelength detuning for bare $\operatorname{SiN}$ waveguides $(\mathrm{N}=0, \mathrm{~L}=0 \mathrm{~mm})$ and hybrid waveguides with both uniformly coated $(N=1, L=20 \mathrm{~mm})$ and patterned $(N=1, L=10 \mathrm{~mm}$ and $N=10, L=1 \mathrm{~mm})$ GO films. The pump wavelength is fixed at $1550 \mathrm{~nm}$ and the signal wavelength was detuned around $1550 \mathrm{~nm}$. (c) Evolutions of idler power along the hybrid waveguides with different $L$ when $N=2$. In (a) - (c), $L 0=0 \mathrm{~mm}$, $\mathrm{W}=1600 \mathrm{~nm}$, and $\mathrm{H}=660 \mathrm{~nm}$ 